\title{
Planar Coil Optimization in a Magnetically Shielded Cylinder
}

\author{
M. Packer®, ${ }^{1}$ P.J. Hobson $\odot,{ }^{1}$ N. Holmes $\odot,{ }^{1,2}$ J. Leggett, ${ }^{1,2}$ P. Glover, ${ }^{1,2}$ M.J. Brookes, ${ }^{1,2}$ \\ R. Bowtell, ${ }^{1,2}$ and T.M. Fromhold ${ }^{1, *}$ \\ ${ }^{1}$ School of Physics and Astronomy, University of Nottingham, Nottingham NG7 2RD, United Kingdom \\ ${ }^{2}$ Sir Peter Mansfield Imaging Centre, School of Physics and Astronomy, University of Nottingham, \\ Nottingham NG7 2RD, United Kingdom
}

(Received 18 January 2021; revised 5 May 2021; accepted 13 May 2021; published 2 June 2021)

\begin{abstract}
Hybrid magnetic shields with both active field generating components and high-permeability magnetic shielding are increasingly needed for various technologies and experiments that require precisioncontrolled magnetic field environments. However, the fields generated by the active components interact with the passive magnetic shield, distorting the desired field profiles. Consequently, optimization of the active components needed to generate user-specified target fields must include coupling to the highpermeability passive components. Here, we consider the optimization of planar active systems, on which an arbitrary static current flows, coupled to a closed high-permeability cylindrical shield. We modify the Green's function for the magnetic vector potential to match boundary conditions on the shield's interior surface, enabling us to construct an inverse optimization problem to design planar coils that generate user-specified magnetic fields inside high-permeability shields. We validate our methodology by designing two biplanar hybrid active-passive systems, which generate a constant transverse field, $\mathbf{B}=\hat{\mathbf{x}}$, and a linear field gradient, $\mathbf{B}=(-x \hat{\mathbf{x}}-y \hat{\mathbf{y}}+2 z \hat{\mathbf{z}})$, respectively. For both systems, the inverse-optimized magnetic field profiles agree well with forward numerical simulations. Our design methodology is accurate and flexible, facilitating the miniaturization of high-performance hybrid magnetic field generating technologies with strict design constraints and spatial limitations.
\end{abstract}

DOI: 10.1103/PhysRevApplied.15.064006

\section{INTRODUCTION}

Tailored, high-precision, low magnetic field environments are required for many applications, devices, and experiments. Examples include magnetic field control in quantum sensing of gravity for underground surveying and mapping [1-4], magnetic field cancelation for atomic magnetometry [5] with applications in medical imaging such as magnetoencephalography [6-9] and neonatal and fetal magnetocardiography [10-12], and noise suppression in fundamental physics experiments [13-15]. Usually, these systems are enclosed by a shield formed from highpermeability material, which reduces stray external magnetic fields that can limit the accuracy and sensitivity of the measurements. In particular, cylindrical shield geometries are often used because the dimensions and spacing of multiple cylindrical shield layers can be optimized to generate a large interior shielded region [16,17]. The magnetic field interior to the shield can then be adjusted by active field generating components to either cancel background fields further or to define a specific field environment. However, the surrounding passive shielding material deforms

*mark.fromhold@nottingham.ac.uk the magnetic field profiles generated by the active components, making it hard to design wire patterns that accurately generate specified target magnetic field profiles [18].

Boundary element methods (BEMs) can be used to optimize magnetic fields generated by surface currents on a triangular mesh [19-22] to generate arbitrary target magnetic fields. BEMs are extremely powerful and flexible since they can be used to define active systems with complex geometries inside passive shields. They are, however, limited by computational power with results depending on mesh size and on the distance of the active components from the shielding material. Alternatively, analytical methods for optimizing hybrid active-passive systems are advantageous since they provide intuition and understanding of how magnetic fields are distorted by the presence of high-permeability material. Analytical formulations also provide a fast and efficient route to determine the best system for generating a bespoke user-specified magnetic field profile.

Currently, analytical models for hybrid active-passive systems are restricted to a limited number of scenarios. Simple discrete coil geometries have been formulated in cylindrical high-permeability magnetic shields, where the magnetic field is decomposed into azimuthal Fourier modes [23-25] and matched at the shield boundary. 
Planar high-permeability materials have been incorporated into optimization procedures using the method of mirror images [26,27] to determine the total magnetic field generated by a static current source inside a magnetically shielded room [28-31]. Similar work in magnetic resonance imaging has investigated the interaction of switched magnetic field gradients with high-conductivity materials used for passive shielding [32]. These formulations incorporate the effect of the shielding material by explicitly solving Maxwell's equations by matching the required boundary conditions on the surface interface to find the total magnetic field generated by the system. More recently, solutions for the interaction between a static current source and a finite-length closed cylindrical high-permeability shield have been formulated for the specific case of cylindrical coaxial surface current geometries [33]. The boundary conditions at the shield's surface were approximated to those of a perfect magnetic conductor and the response of the shield was calculated by matching the azimuthal Fourier modes and using the method of mirror images. The magnetic field generated by the entire system was then calculated in terms of the initial current source and used in conjunction with a least squares optimization to determine globally optimal cylindrical currents to generate arbitrary magnetic field profiles. However, coaxial cylindrical coils are not appropriate to all systems because of length and optical access limitations.

In this work, by applying the same approach as Packer et al. [33], we analytically determine the effect of a finitelength closed cylindrical high-permeability shield on the magnetic field generated by an arbitrary static current distribution on an interior circular plane, oriented perpendicular to the axis of the cylinder. We calculate the effect of the high-permeability cylinder on the magnetic field produced by current flow on the plane, which enables us to determine optimal current paths to generate user-specified target fields inside the cylinder. We first derive the vector potential generated by an arbitrary planar current source. Then, we calculate a pseudocurrent density induced on the cylindrical surface of the high-permeability material in response to the planar current source, and, hence, derive a Green's function for our system. Finally, we implement a Fourier decomposition of the current paths to calculate the total magnetic field in terms of a set of weighted Fourier coefficients. This formulation allows the incorporation of a quadratic optimization procedure to determine globally optimal designs that generate user-specified target magnetic field profiles. Through the use of this optimization procedure, we design two example biplanar coil systems optimized for operation inside a finite-length closed highpermeability cylindrical magnetic shield. In both cases, we confirm that our analytical model agrees well with the result of numerical finite element simulations. Our work extends the range of coil geometries that can be efficiently optimized to generate static user-specified target magnetic fields in the presence of high-permeability materials, expanding design flexibility for systems that require precision-controlled magnetic field environments.

\section{THEORY}

In Ref. [33] the interaction between an arbitrary static current flow on a cylinder and an exterior finite-length closed high-permeability cylindrical magnetic shield was formulated analytically. The vector potential in free space was related to an arbitrary current on the cylinder $\mathbf{j}_{\mathbf{c}}$, and

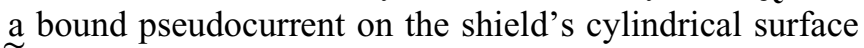
$\widetilde{\mathbf{j}}$, which is equivalent to the induced magnetization of the shield, resulting in

$$
\nabla^{2} \mathbf{A}=-\mu_{0}\left(\mathbf{j}_{\mathbf{c}}+\widetilde{\mathbf{j}}\right),
$$

with integral solution in terms of an arbitrary current density

$$
\mathbf{A}(\mathbf{r})=\mu_{0} \int_{r^{\prime}} d^{3} \mathbf{r}^{\prime} G\left(\mathbf{r}, \mathbf{r}^{\prime}\right) \mathbf{j}\left(\mathbf{r}^{\prime}\right)
$$

where $G\left(\mathbf{r}, \mathbf{r}^{\prime}\right)$ is the associated Green's function for the system [26]. It was then shown that the high-permeability magnetic shield could be assumed to be a perfect magnetic conductor with minimal error, meaning a combination of methods could be used to satisfy the boundary conditions at the shield's surface. From this, the total magnetic field generated by the entire system could be efficiently calculated in terms of the initial current source. An orthogonal current density basis was then used in conjunction with a least squares optimization to determine globally optimal current flow patterns in a cylindrical basis. The subsequent example designs were verified using numerical finite element software, showing deviations from the analytical model of $\mathcal{O}\left(\mu^{-1}\right)$, in line with previous findings [34,35]. Here, we use the same methodology to find optimal current flow patterns on a planar basis interior to a finite-length closed high-permeability cylindrical magnetic shield.

We consider a closed cylindrical magnetic shield surrounded by free space, as shown in Fig. 1. This cylinder has relative permeability, $\mu_{r} \gg 1$, radius $\rho_{s}$, and length $L_{s}$, with planar end caps located at $z= \pm L_{s} / 2$. Inside this cylinder, an arbitrary current flows on a circular planar surface of radius $\rho_{c}<\rho_{s}$, centered on the $z$ axis and lying in the $z=z^{\prime}$ plane where $\left|z^{\prime}\right|<L_{s} / 2$.

The boundary conditions on the surface of the shield, illustrated in Fig. 1, are approximated to that of a perfect magnetic conductor

$$
\left.B_{\rho}\right|_{z= \pm L_{s} / 2}=0,\left.\quad B_{\phi}\right|_{z= \pm L_{s} / 2, \rho=\rho_{s}}=0,\left.\quad B_{z}\right|_{\rho=\rho_{s}}=0,
$$

provided the material permeability and thickness are sufficiently large [33]. 


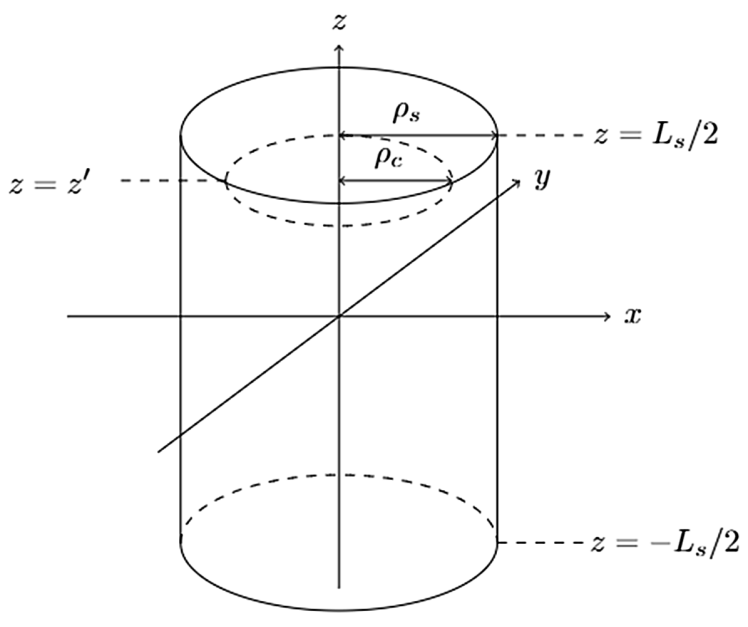

FIG. 1. A cylindrical hollow high-permeability magnetic shield of length $L_{s}$, radius $\rho_{s}$, and with planar end caps located at $z= \pm L_{s} / 2$ encloses an interior circular current source (bounded by the upper dashed curve) of radius $\rho_{c}<\rho_{s}$, which lies in the $z=z^{\prime}$ plane.

The vector potential generated by a current source in cylindrical coordinates can be expressed as

$$
\begin{aligned}
A_{\rho}(\mathbf{r})= & \mu_{0} \int_{r^{\prime}} d^{3} \mathbf{r}^{\prime} G\left(\mathbf{r}, \mathbf{r}^{\prime}\right)\left[j_{\rho}\left(\mathbf{r}^{\prime}\right) \cos \left(\phi-\phi^{\prime}\right)\right. \\
& \left.+j_{\phi}\left(\mathbf{r}^{\prime}\right) \sin \left(\phi-\phi^{\prime}\right)\right], \\
A_{\phi}(\mathbf{r})= & -\mu_{0} \int_{r^{\prime}} d^{3} \mathbf{r}^{\prime} G\left(\mathbf{r}, \mathbf{r}^{\prime}\right)\left[j_{\rho}\left(\mathbf{r}^{\prime}\right) \sin \left(\phi-\phi^{\prime}\right)\right. \\
& \left.-j_{\phi}\left(\mathbf{r}^{\prime}\right) \cos \left(\phi-\phi^{\prime}\right)\right], \\
A_{z}(\mathbf{r})= & \mu_{0} \int_{r^{\prime}} d^{3} \mathbf{r}^{\prime} G\left(\mathbf{r}, \mathbf{r}^{\prime}\right) j_{z}\left(\mathbf{r}^{\prime}\right) .
\end{aligned}
$$

Since there is no current flow in the $z$ direction for a planar current source perpendicular to the axis of the cylindrical shield, the continuity equation can be used to express the planar current flow in terms of a single scalar streamfunction defined on the planar surface

$$
j_{\rho}\left(\mathbf{r}^{\prime}\right)=\frac{1}{\rho^{\prime}} \frac{\partial \varphi\left(\mathbf{r}^{\prime}\right)}{\partial \phi^{\prime}}, \quad j_{\phi}\left(\mathbf{r}^{\prime}\right)=-\frac{\partial \varphi\left(\mathbf{r}^{\prime}\right)}{\partial \rho^{\prime}}
$$

where $\varphi\left(\mathbf{r}^{\prime}\right)=\varphi\left(\rho^{\prime}, \phi^{\prime}\right)$. To exploit the radial symmetries of the system, we choose to decompose the Green's function in cylindrical coordinates in terms of Bessel functions of the first kind,

$$
\begin{aligned}
G\left(\mathbf{r}, \mathbf{r}^{\prime}\right)= & \frac{1}{4 \pi} \sum_{m=-\infty}^{\infty} e^{i m\left(\phi-\phi^{\prime}\right)} \\
& \times \int_{0}^{\infty} d k e^{-k\left|z-z^{\prime}\right|} J_{m}(k \rho) J_{m}\left(k \rho^{\prime}\right),
\end{aligned}
$$

allowing the vector potential to be expressed in terms of cylindrical harmonics defined on a circular plane. Using Eqs. (4)-(8), we cast the vector potential in a simplified form

$$
\begin{aligned}
& A_{\rho}(\mathbf{r})=\frac{i \mu_{0}}{2 \rho} \sum_{m=-\infty}^{\infty} e^{i m \phi} \int_{0}^{\infty} d k m e^{-k\left|z-z^{\prime}\right|} J_{m}(k \rho) \varphi^{m}(k), \\
& A_{\phi}(\mathbf{r})=-\frac{\mu_{0}}{2} \sum_{m=-\infty}^{\infty} e^{i m \phi} \int_{0}^{\infty} d k k e^{-k\left|z-z^{\prime}\right|} J_{m}^{\prime}(k \rho) \varphi^{m}(k),
\end{aligned}
$$

$$
A_{z}(\mathbf{r})=0
$$

where $J_{m}^{\prime}(z)$ is the derivative of $J_{m}(z)$ with respect to $z$, and $\varphi^{m}(k)$ is defined as the $m$ th order Hankel transform

$$
\varphi^{m}(k)=\frac{1}{2 \pi} \int_{0}^{\infty} d \rho^{\prime} \int_{0}^{2 \pi} d \phi^{\prime} e^{-i m \phi^{\prime}} \rho^{\prime} J_{m}\left(k \rho^{\prime}\right) \varphi\left(\rho^{\prime}, \phi\right) .
$$

We now consider the vector potential generated by the magnetic shield. We seek a Fourier representation of the bound pseudocurrent density, $\tilde{\mathbf{j}}=\tilde{j_{\phi}}\left(\phi^{\prime}, z^{\prime}\right) \hat{\boldsymbol{\phi}}+\tilde{j_{z}}\left(\phi^{\prime}, z^{\prime}\right) \hat{\mathbf{z}}$, which satisfies the boundary condition over the entire domain of the shield. In particular, we wish to equate the shared azimuthal Fourier modes at the radial boundary of the shield cylinder. This is achieved using a combination of methods that must be applied sequentially, since each method satisfies the condition at an orthogonal boundary. The radial condition is satisfied by equating the magnetic field generated by the cylindrical pseudocurrent density and planar current flow, generating a relation between the response of an infinite cylindrical shield and the initial current source. Then, the boundary condition at the end caps can simultaneously be satisfied by applying the method of mirror images [26]. These methods can be combined because the infinite pseudocurrent density and planar current flow are spatially orthogonal to the end caps, meaning that any reflections generated by the application of the method of mirror images continue to satisfy the radial condition. The components of the vector potential generated by a pseudocurrent density induced on an infinite cylinder [32] in the region $\rho<\rho_{s}$ are given by

$$
\begin{aligned}
A_{\rho}(\rho, \phi, z)= & -\frac{i \mu_{0} \rho_{s}}{4 \pi} \sum_{m=-\infty}^{\infty} \int_{-\infty}^{\infty} d k e^{i m \phi} e^{i k z} \\
& \times\left[I_{m-1}(|k| \rho) K_{m-1}\left(|k| \rho_{s}\right)\right. \\
& \left.-I_{m+1}(|k| \rho) K_{m+1}\left(|k| \rho_{s}\right)\right] \tilde{j}_{\phi}^{m}(k),
\end{aligned}
$$




$$
\begin{aligned}
A_{\phi}(\rho, \phi, z)= & \frac{\mu_{0} \rho_{s}}{4 \pi} \sum_{m=-\infty}^{\infty} \int_{-\infty}^{\infty} d k e^{i m \phi} e^{i k z} \\
& \times\left[I_{m-1}(|k| \rho) K_{m-1}\left(|k| \rho_{s}\right)\right. \\
+ & \left.I_{m+1}(|k| \rho) K_{m+1}\left(|k| \rho_{s}\right)\right] \tilde{j}_{\phi}^{m}(k), \\
A_{z}(\rho, \phi, z)= & \frac{\mu_{0} \rho_{s}}{2 \pi} \sum_{m=-\infty}^{\infty} \int_{-\infty}^{\infty} d k e^{i m \phi} e^{i k z} \\
& \times I_{m}(|k| \rho) K_{m}\left(|k| \rho_{s}\right) \tilde{j}_{z}^{m}(k),
\end{aligned}
$$

where the Fourier transforms of the pseudocurrents are defined by

$$
\begin{aligned}
& j_{\phi}^{m}(k)=\frac{1}{2 \pi} \int_{0}^{2 \pi} d \phi^{\prime} e^{-i m \phi^{\prime}} \int_{-\infty}^{\infty} d z^{\prime} e^{-i k z^{\prime}} \tilde{j_{\phi}}\left(\phi^{\prime}, z^{\prime}\right), \\
& j_{z}^{m}(k)=\frac{1}{2 \pi} \int_{0}^{2 \pi} d \phi^{\prime} e^{-i m \phi^{\prime}} \int_{-\infty}^{\infty} d z^{\prime} e^{-i k z^{\prime}} \tilde{j}_{z}\left(\phi^{\prime}, z^{\prime}\right)
\end{aligned}
$$

The corresponding inverse transforms are given by

$$
\begin{aligned}
& \tilde{j_{\phi}}\left(\phi^{\prime}, z^{\prime}\right)=\frac{1}{2 \pi} \sum_{m=-\infty}^{\infty} \int_{-\infty}^{\infty} d k e^{i m \phi^{\prime}} e^{i k z^{\prime}} j_{\phi}^{m}(k), \\
& \tilde{j_{z}}\left(\phi^{\prime}, z^{\prime}\right)=\frac{1}{2 \pi} \sum_{m=-\infty}^{\infty} \int_{-\infty}^{\infty} d k e^{i m \phi^{\prime}} e^{i k z^{\prime}} j_{z}^{m}(k) .
\end{aligned}
$$

Therefore, adding the contributions from the planar current flow, Eqs. (9)-(10), and the infinite pseudocurrent density, Eqs. (13)-(15), while using Eqs. (16)-(19), and applying the method of mirror images for two infinitely large parallel planes, we can write the total magnetic field generated by the system in the region $\rho<\rho_{S}$ as

$$
\begin{aligned}
B_{\rho}(\rho, \phi, z)= & -\frac{\mu_{0}}{2 \pi} \sum_{p=-\infty}^{\infty} \sum_{m=-\infty}^{\infty} e^{i m \phi} \\
& \times\left[\int_{0}^{\infty} d k k^{2} \frac{z-(-1)^{p} z^{\prime}+p L_{s}}{\left|z-(-1)^{p} z^{\prime}+p L_{s}\right|}\right. \\
& \times e^{-k\left|z-(-1)^{p} z^{\prime}+p L_{s}\right|} J_{m}^{\prime}(k \rho) \varphi^{m}(k) \\
& \left.-\frac{i \rho_{s}}{\pi} \int_{-\infty}^{\infty} d k k e^{i k z} I_{m}^{\prime}(|k| \rho) K_{m}^{\prime}\left(|k| \rho_{s}\right) j_{\phi}^{m p}(k)\right],
\end{aligned}
$$

$$
\begin{aligned}
B_{\phi}(\rho, \phi, z)= & -\frac{i \mu_{0}}{2 \rho} \sum_{p=-\infty}^{\infty} \sum_{m=-\infty}^{\infty} m e^{i m \phi} \\
& \times\left[\int_{0}^{\infty} d k k \frac{z-(-1)^{p} z^{\prime}+p L_{s}}{\left|z-(-1)^{p} z^{\prime}+p L_{s}\right|}\right. \\
& \times e^{-k\left|z-(-1)^{p} z^{\prime}+p L_{s}\right|} J_{m}(k \rho) \varphi^{m}(k) \\
& \left.-\frac{i \rho_{s}}{\pi} \int_{-\infty}^{\infty} d k \frac{|k|}{k} e^{i k z} I_{m}(|k| \rho) K_{m}^{\prime}\left(|k| \rho_{s}\right) j_{\phi}^{m p}(k)\right], \\
B_{z}(\rho, \phi, z)= & \frac{\mu_{0}}{2} \sum_{p=-\infty}^{\infty} \sum_{m=-\infty}^{\infty} e^{i m \phi} \\
& \times\left[\int_{0}^{\infty} d k k^{2} e^{-k\left|z-(-1)^{p} z^{\prime}+p L_{s}\right|} J_{m}(k \rho) \varphi^{m}(k)\right. \\
& \left.-\frac{\rho_{s}}{\pi} \int_{-\infty}^{\infty} d k|k| e^{i k z} I_{m}(|k| \rho) K_{m}^{\prime}\left(|k| \rho_{s}\right) j_{\phi}^{m p}(k)\right],
\end{aligned}
$$

where $j_{\phi}^{m p}(k)$ is the $p$ th reflected Fourier-transformed azimuthal pseudocurrent density induced on the cylindrical surface of the magnetic shield with $I_{m}^{\prime}(z)$ and $K_{m}^{\prime}(z)$ defined as the derivatives with respect to $z$ of the modified Bessel functions of the first and second kinds, $I_{m}(z)$ and $K_{m}(z)$, respectively. By applying the boundary condition at the radial surface, Eq. (3), we can match the shared $m$ th azimuthal Fourier mode generated by each $p$ th reflected pseudocurrent and streamfunction, resulting in the relation

$$
\begin{aligned}
& \int_{-\infty}^{\infty} d k|k| e^{i k z} I_{m}\left(|k| \rho_{s}\right) K_{m}^{\prime}\left(|k| \rho_{s}\right) j_{\phi}^{m p}(k) \\
& \quad=\frac{\pi}{\rho_{s}} \int_{0}^{\infty} d k k^{2} e^{-k\left|z-(-1)^{p} z^{\prime}+p L_{s}\right|} J_{m}\left(k \rho_{s}\right) \varphi^{m}(k) .
\end{aligned}
$$

Physically, due to the formulation of the response in terms of a pseudocurrent density, there must be a unique solution that is independent of the axial position that satisfies the boundary condition over the infinite domain of the cylindrical shield. Therefore, we perform an inverse Fourier transform with respect to $z$ to generate an integral representation of the $p$ th reflected Fourier pseudocurrent density, $j_{\phi}^{m p}(k)$, in terms of the $m$ th-order Hankel transform of the streamfunction defined on the planar surface, $\varphi^{m}(k)$,

$$
\begin{aligned}
j_{\phi}^{m p}(k)= & \frac{e^{-i k\left[(-1)^{p} z^{\prime}+p L_{s}\right]}}{\rho_{s}|k| I_{m}\left(|k| \rho_{s}\right) K_{m}^{\prime}\left(|k| \rho_{s}\right)} \\
& \times \int_{0}^{\infty} d \tilde{k} \frac{\tilde{k}^{3}}{\tilde{k}^{2}+k^{2}} J_{m}\left(\tilde{k} \rho_{s}\right) \varphi^{m}(\tilde{k}) .
\end{aligned}
$$

This expression for $j_{\phi}^{m p}(k)$ can now be substituted into Eqs. (20)-(22) to determine the total magnetic field in 
terms of $\varphi^{m}(k)$. Next, we must choose an appropriate expansion of the streamfunction, $\varphi\left(\rho^{\prime}, \phi^{\prime}\right)$. Although the choice of orthogonal basis for the expansion of the streamfunction is somewhat arbitrary, a choice of basis that considers the symmetries between the Hankel transform, coordinate system, and the integral representation of the pseudocurrent yields a simpler solution. Here, we choose to decompose the radial component of the planar current flow into a Fourier-Bessel series while using a Fourier series representation of the azimuthal dependence,

$$
\varphi\left(\rho^{\prime}, \phi^{\prime}\right)=\left[H\left(\rho^{\prime}\right)-H\left(\rho-\rho_{c}\right)\right] \rho_{c} \sum_{n=1}^{N} \sum_{m^{\prime}=0}^{M} J_{m^{\prime}}\left(\frac{\rho_{n m^{\prime}} \rho^{\prime}}{\rho_{c}}\right)\left[W_{n m^{\prime}} \cos \left(m^{\prime} \phi^{\prime}\right)+Q_{n m^{\prime}} \sin \left(m^{\prime} \phi^{\prime}\right)\right],
$$

where $\left(W_{n m^{\prime}}, Q_{n m^{\prime}}\right)$ are Fourier coefficients and $\rho_{n m^{\prime}}$ is the $n$th zero of the $m^{\prime}$ th Bessel function of the first kind, $J_{m^{\prime}}\left(\rho_{n m^{\prime}}\right)=0$. Therefore, using Eqs. (20)-(22), (24), and (25), the total magnetic field generated by an arbitrary current flow on the planar surface inside the closed finite high-permeability cylinder can be written as

$$
\begin{aligned}
& B_{\rho}(\rho, \phi, z)=\frac{\mu_{0} \rho_{c}^{3}}{2} \sum_{n=1}^{N} \sum_{m=0}^{M} \rho_{n m} J_{m}^{\prime}\left(\rho_{n m}\right)\left[W_{n m} \cos (m \phi)+Q_{n m} \sin (m \phi)\right] B_{\rho}^{n m}(\rho, z), \\
& B_{\phi}(\rho, \phi, z)=\frac{\mu_{0} \rho_{c}^{3}}{2 \rho} \sum_{n=1}^{N} \sum_{m=0}^{M} m \rho_{n m} J_{m}^{\prime}\left(\rho_{n m}\right)\left[W_{n m} \sin (m \phi)-Q_{n m} \cos (m \phi)\right] B_{\phi}^{n m}(\rho, z), \\
& B_{z}(\rho, \phi, z)=\frac{\mu_{0} \rho_{c}^{3}}{2} \sum_{n=1}^{N} \sum_{m=0}^{M} \rho_{n m} J_{m}^{\prime}\left(\rho_{n m}\right)\left[W_{n m} \cos (m \phi)+Q_{n m} \sin (m \phi)\right] B_{z}^{n m}(\rho, z),
\end{aligned}
$$

where

$$
\begin{aligned}
& B_{\rho}^{n m}(\rho, z)=-\int_{0}^{\infty} d k k^{2} \sigma\left(k ; z, z^{\prime}, L_{s}\right) \frac{J_{m}^{\prime}(k \rho) J_{m}\left(k \rho_{c}\right)}{k^{2} \rho_{c}^{2}-\rho_{n m}^{2}}-\sum_{p=1}^{\infty} \tilde{p}|\tilde{p}| \lambda_{p}\left(z, z^{\prime}, L_{s}\right) \frac{I_{m}^{\prime}(|\tilde{p}| \rho) I_{m}\left(|\tilde{p}| \rho_{c}\right) K_{m}\left(|\tilde{p}| \rho_{s}\right)}{I_{m}\left(|\tilde{p}| \rho_{s}\right)\left(|\tilde{p}|^{2} \rho_{c}^{2}+\rho_{n m}^{2}\right)} \\
& B_{\phi}^{n m}(\rho, z)=\int_{0}^{\infty} d k k \sigma\left(k ; z, z^{\prime}, L_{s}\right) \frac{J_{m}(k \rho) J_{m}\left(k \rho_{c}\right)}{k^{2} \rho_{c}^{2}-\rho_{n m}^{2}}+\sum_{p=1}^{\infty} \tilde{p} \lambda_{p}\left(z, z^{\prime}, L_{s}\right) \frac{I_{m}(|\tilde{p}| \rho) I_{m}\left(|\tilde{p}| \rho_{c}\right) K_{m}\left(|\tilde{p}| \rho_{s}\right)}{I_{m}\left(|\tilde{p}| \rho_{s}\right)\left(|\tilde{p}|^{2} \rho_{c}^{2}+\rho_{n m}^{2}\right)} \\
& B_{z}^{n m}(\rho, z)=\int_{0}^{\infty} d k k^{2} \gamma\left(k ; z, z^{\prime}, L_{s}\right) \frac{J_{m}(k \rho) J_{m}\left(k \rho_{c}\right)}{k^{2} \rho_{c}^{2}-\rho_{n m}^{2}}-\sum_{p=1}^{\infty} \tilde{p}^{2} \tau_{p}\left(z, z^{\prime}, L_{s}\right) \frac{I_{m}(|\tilde{p}| \rho) I_{m}\left(|\tilde{p}| \rho_{c}\right) K_{m}\left(|\tilde{p}| \rho_{s}\right)}{I_{m}\left(|\tilde{p}| \rho_{s}\right)\left(|\tilde{p}|^{2} \rho_{c}^{2}+\rho_{n m}^{2}\right)}
\end{aligned}
$$

and

$$
\begin{aligned}
\gamma\left(k ; z, z^{\prime}, L_{s}\right) & =e^{-k\left|z-z^{\prime}\right|}+\frac{2}{e^{2 k L_{s}}-1}\left\{e^{k L_{s}} \cosh \left[k\left(z+z^{\prime}\right)\right]+\cosh \left[k\left(z-z^{\prime}\right)\right]\right\}, \\
\sigma\left(k ; z, z^{\prime}, L_{s}\right) & =\frac{z-z^{\prime}}{\left|z-z^{\prime}\right|} e^{-k\left|z-z^{\prime}\right|}-\frac{2}{e^{2 k L_{s}}-1}\left\{e^{k L_{s}} \sinh \left[k\left(z+z^{\prime}\right)\right]+\sinh \left[k\left(z-z^{\prime}\right)\right]\right\}, \\
\lambda_{p}\left(z, z^{\prime}, L_{s}\right) & =\frac{2}{L_{s}}\left\{(-1)^{p} \sin \left[\tilde{p}\left(z+z^{\prime}\right)\right]+\sin \left[\tilde{p}\left(z-z^{\prime}\right)\right]\right\}, \\
\tau_{p}\left(z, z^{\prime}, L_{s}\right) & =\frac{2}{L_{s}}\left\{(-1)^{p} \cos \left[\tilde{p}\left(z+z^{\prime}\right)\right]+\cos \left[\tilde{p}\left(z-z^{\prime}\right)\right]\right\},
\end{aligned}
$$

with $\tilde{p}=p \pi / L_{s}$. A full derivation of these expressions is given in Appendix A. Here, as in Ref. [33], we solve for the unknown Fourier coefficients, $\left(W_{n m}, Q_{n m}\right)$, using a least squares minimization with the addition of the power as the regularization parameter. The power $P$ dissipated by a circular planar current source of thickness $t$ and resistivity $\varrho$ is given by 


$$
P=\frac{\varrho}{t} \int_{0}^{\rho_{c}} d \rho^{\prime} \rho^{\prime} \int_{0}^{2 \pi} d \phi^{\prime}\left|J_{\rho}\left(\rho^{\prime}, \phi^{\prime}\right)\right|^{2}+\left|J_{\phi}\left(\rho^{\prime}, \phi^{\prime}\right)\right|^{2} .
$$

Substituting Eq. (25) into Eq. (7) and then Eq. (36), and integrating over the planar surface we find that, for $m=0$,

$$
P=\frac{\varrho}{t} \pi \rho_{c}^{2} \sum_{n=1}^{N} W_{n 0}^{2} \rho_{n 0}^{2} J_{1}\left(\rho_{n 0}\right)^{2},
$$

and, for $m \in \mathbb{Z}^{+}$,

$$
\begin{aligned}
P= & \frac{\varrho}{t} \pi \rho_{c}^{2} \sum_{n=1}^{N} \sum_{m=1}^{M}\left(W_{n m}^{2}+Q_{n m}^{2}\right)\left(\frac{\rho_{n m}}{2}\right)^{2 m} \frac{1}{m !(m-1) !}\left[{ }_{2} \tilde{F}_{3}\left(m, m+\frac{1}{2} ; m+1, m+1,2 m+1 ;-\rho_{n m}^{2}\right)\right. \\
& \left.-\frac{\rho_{n m}^{2}}{2(m+1)^{2}}{ }_{3} \tilde{F}_{4}\left(m+\frac{1}{2}, m+1, m+1 ; m, m+2, m+2,2 m+1 ;-\rho_{n m}^{2}\right)\right],
\end{aligned}
$$

where ${ }_{i} \tilde{F}_{j}$ is the regularized hypergeometric function; see Appendix B for a full derivation. The cost function for the least squares minimization is thus given by

$$
\Phi=\sum_{k}^{K}\left[\mathbf{B}^{\text {desired }}\left(\mathbf{r}_{k}\right)-\mathbf{B}\left(\mathbf{r}_{k}\right)\right]^{2}+\beta P,
$$

where $\beta$ is a weighting parameter chosen to adjust the physical constraints of the system. The cost function is minimized using a least squares fitting to calculate the optimal Fourier coefficients to generate the desired magnetic field at $K$ target points. The minimization is achieved by taking the differential of the cost function with respect to the Fourier coefficients,

$$
\frac{\partial \Phi}{\partial W_{i j}}=0, \quad \frac{\partial \Phi}{\partial Q_{i j}}=0, \quad \text { for } i \geq 1, j \geq 0,
$$

which enables the optimal Fourier coefficients to be found for any given physical target magnetic field profile through matrix inversion. The inversion process yields the optimal continuous streamfunction defined on the planar surface for a finite number of Fourier coefficients. The number of Fourier coefficients should be chosen and the streamfunction discretized in accordance with previous work [33]. Other optimization methods, such as linear programming, could be used to determine optimal designs to allow for more direct constraints on the field fidelity at specific target points [21,22]. Alternatively, the optimal Fourier coefficients for a specific target region could be found using a numerical procedure such as a particle swarm optimization [36].

\section{RESULTS}

We now verify our analytical model by designing hybrid active-passive systems composed of biplanar coils inside a closed high-permeability magnetic shield and compare the resulting magnetic field profiles with forward numerical simulations of each optimized system. We consider two distinct systems, each containing current confined to two disks of radius $\rho_{c}=0.45 \mathrm{~m}$ and symmetrically placed at $z^{\prime}= \pm 0.45 \mathrm{~m}$. Both systems are interior to a perfect closed cylindrical magnetic shield of radius $\rho_{s}=0.5 \mathrm{~m}$ and length $L_{s}=1 \mathrm{~m}$ centered on the origin, as shown in Fig. 2. The first system is designed to generate a constant transverse field, $\mathbf{B}=B_{0} \hat{\mathbf{x}}$, and the second system creates a linear field gradient, $\mathbf{B}=(-x \hat{\mathbf{x}}-y \hat{\mathbf{y}}+2 z \hat{\mathbf{z}})$, within an optimization region defined by $-z^{\prime} / 2 \leq z \leq z^{\prime} / 2$ and $0 \leq \rho \leq \rho_{c} / 4$. The magnetic field profiles chosen, i.e., the uniform transverse and linear gradient fields, are examples of tesseral $(m \neq 0)$ and zonal $(m=0)$ harmonics,

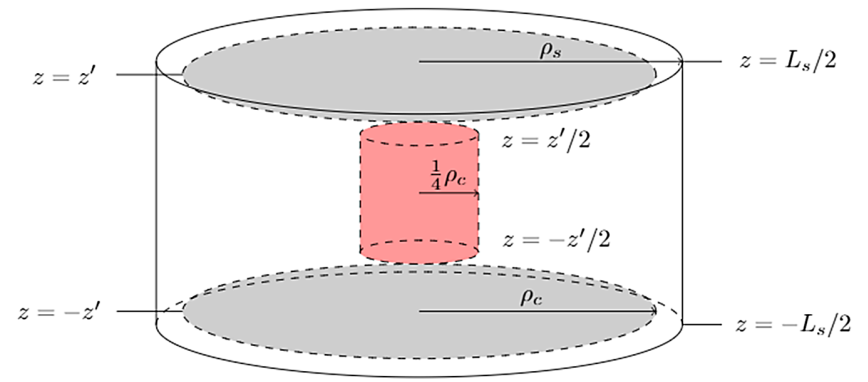

FIG. 2. Schematic diagram showing the areas occupied by the biplanar coils (light gray) inside a cylindrical closed high-permeability magnetic shield (black outline). The highpermeability shield is of length $L_{s}=1 \mathrm{~m}$ and radius $\rho_{s}=0.5 \mathrm{~m}$, with planar end caps located at $z= \pm L_{s} / 2$. The shield encloses interior conducting planes of radius $\rho_{c}=0.45 \mathrm{~m}$ that are located at $z^{\prime}= \pm 0.45 \mathrm{~m}$. The optimization region (red) is bounded along the $z$ axis between the coil planes with top and bottom positions $z= \pm z^{\prime} / 2$, respectively, and extends radially between $\rho=\left[0, \rho_{c} / 4\right]$. 

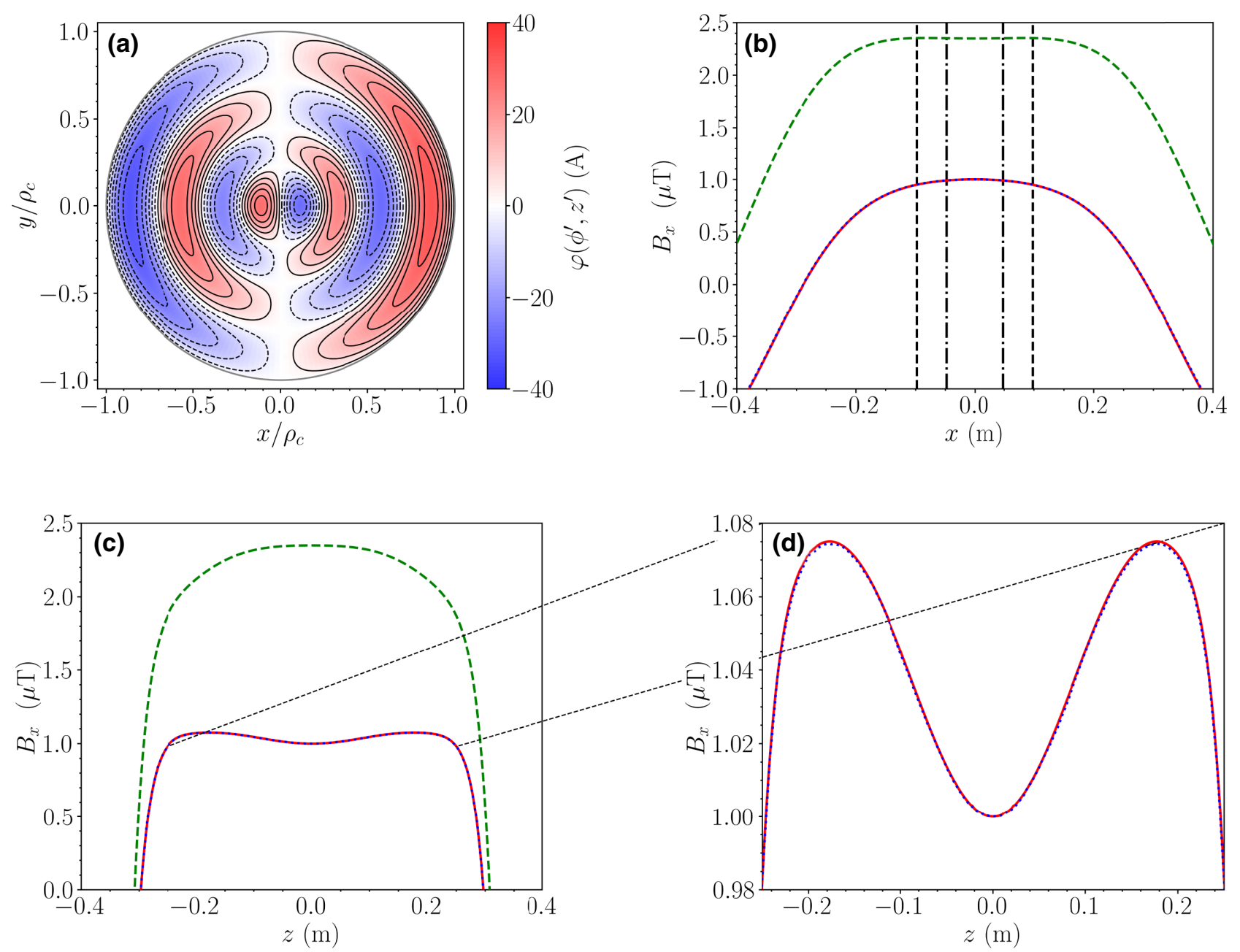

FIG. 3. Wire layouts (a) and performance (b)-(d) of a hybrid active-passive system optimized to generate a constant transverse magnetic field, $B_{x}$. Current flows on the surface of two disks of radius $\rho_{c}=0.45 \mathrm{~m}$, which are separated symmetrically from the origin and lie at $z^{\prime}= \pm 0.45 \mathrm{~m}$. The wire layouts are optimized to generate a constant transverse field, $B_{0}=1 \mu \mathrm{T}$, across the cylinder and normal to its axis of symmetry. The current-carrying planes are placed symmetrically inside a perfect closed magnetic shield of radius $\rho_{s}=0.5 \mathrm{~m}$ and length $L_{s}=1 \mathrm{~m}$ and the magnetic field is optimized between $\rho=\left[0, \rho_{c} / 4\right]$ and $z= \pm z^{\prime} / 2$, as shown in Fig. 2. The least squares optimization is performed with parameters $N=50, M=1, \beta=1.77 \times 10^{-9} \mathrm{~T}^{2} / \mathrm{W}, t=0.5 \mathrm{~mm}$, and $\rho=1.68 \times 10^{-8} \Omega \mathrm{m}$. (a) Color map of the optimal current streamfunction calculated for the upper current-carrying plane in Fig. 2. Blue and red shaded regions correspond to current counterflows and their intensity shows the streamfunction magnitude from low (white) to high (intense color). Solid and dashed black curves represent discrete wires with opposite senses of current flow, approximating the current continuum with $N_{\varphi}=12$ global contour levels. Streamfunction on the lower light gray plane in Fig. 2 is geometrically identical but the current direction is reversed. (b) Transverse magnetic field, $B_{x}$, calculated versus transverse position, $x$, for $y=z=0$, from the current continuum in (a) in three ways: analytically using Eqs. (26)-(28) (solid red curve); numerically using COMSOL Multiphysics version 5.5a, modeling the high-permeability cylinder as a perfect magnetic conductor (blue dotted curve); numerically without the high-permeability cylinder and using the Biot-Savart law with $N_{\varphi}=100$ contour levels (dashed green curve). Black lines enclose the regions where the calculated field deviates from the target field by $5 \%$ (dashed) and $1 \%$ (dash-dot). (c) Transverse magnetic field, $B_{x}$, calculated versus axial position, $z$, for $x=y=0$, from the current continuum in the same three ways as for (b). (d) Enlarged section of (c) showing good agreement between the numerical and analytical results throughout the optimization region.

respectively, which exhibit $m$-fold and complete azimuthal symmetry [37], which facilitates analysis of the shield's particular response to tesseral and zonal harmonic fields generated by planar current sources. The two systems that we consider here are chosen to illustrate targeted magnetic field compensation in situations where compact systems are required, but space inside the central cylindrical cavity of the system is limited by the presence of experimental equipment (e.g., magnetic sensors). Coil designs generating other field profiles, for example using 
combinations of planar coils with varying sizes, can be found in Appendix C. All designs can be replicated using our open-access PYTHON code [38].

In Figs. 3(a) and 5(a), we show the optimized contoured streamfunctions for the constant transverse and linear field gradient systems, respectively. Because of the symmetric placement of the biplanar coil systems and optimized field regions within the shield (see Fig. 2), the magnitudes of the streamfunctions defined on both coils are identical. The current flow directions, however, are opposite, due to the form of the desired fields. Figures 3(b)-3(d) show the transverse magnetic field, $B_{0}=1 \mu \mathrm{T}$, along the $x$ axis and $z$ axis, respectively, generated by the biplanar coil design shown in Fig. 3(a) calculated in three different ways: analytically using Eqs. (26)-(28) (solid red curves); numerically using COMSOL Multiphysics ${ }^{\circledR}$ with the shield treated as a perfect magnetic conductor (blue dotted curves); numerically in free space, i.e., excluding the high-permeability material and evaluating the magnetic fields through the Biot-Savart law for discretized biplanar coils with $N_{\varphi}=100$ (dashed green curve). Furthermore, color maps of the transverse and axial magnetic field components in the $x-z$ plane are presented in Figs. 4(a) and 4(b), respectively, calculated numerically using COMSOL Multiphysics with the shield treated as a perfect magnetic conductor. Similarly, Figs. 5(b) and 5(c) show the field gradients, $d B_{x} / d x=-1 \mu \mathrm{T} / \mathrm{m}$ and $d B_{z} / d z=2 \mu \mathrm{T} / \mathrm{m}$, along the $x$ axis and $z$ axis, respectively, generated by the biplanar coil system shown in Fig. 5(a) in the same three cases with similar color maps of the magnetic field components in the $x-z$ plane shown in Figs. 6(a) and 6(b). For both designs, the analytical field profiles agree well with numerical simulations. The maximum errors between the analytical model and numerical simulation are $0.052 \%$ and $0.043 \%$ for the constant transverse field and the gradient of the linear gradient field, respectively, along the $z$ axis of the optimization region. The error between these results could be decreased by reducing the mesh size at a cost of increased computational effort. Here, the mesh size has been adapted so that the maximum difference between the analytical and numerical results is minimized, in line with previous work [33], and, crucially, is below the error introduced from the perfect magnetic conducting shield approximation $[34,35]$.

To quantify the performance of our optimization procedure, we can analyze the deviations between the magnetic fields generated by our theoretical model and the desired target fields. Examining the fidelity of the fields generated by both systems along the $x$ axis of the optimization region, the maximum absolute deviations from the constant transverse and linear axial gradient fields are $6.78 \%$ and $0.380 \%$, respectively. Along the $z$ axis of the optimization region, the maximum deviations are $7.50 \%$ and $0.306 \%$, respectively. Clearly, the axial field gradient is generated more accurately than the uniform transverse (a)

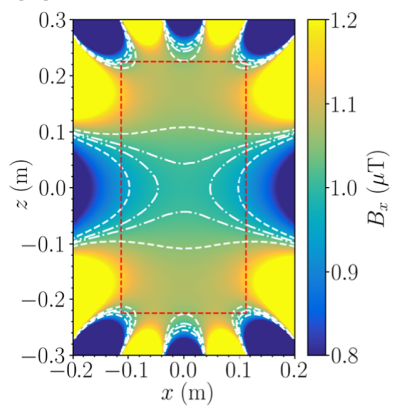

(b)

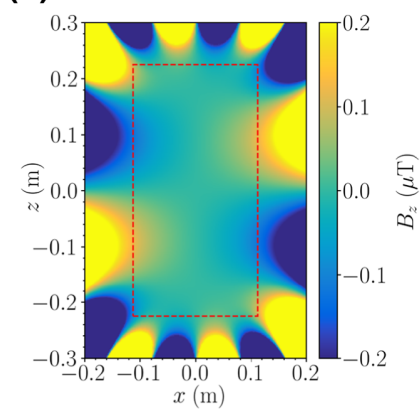

FIG. 4. Color maps showing the magnitude of the magnetic field, in the $x-z$ plane inside a closed finite-length perfect magnetic conductor generated by the active-passive system depicted in Fig. 3: (a) transverse component, $B_{x}$, and (b) axial component, $B_{z}$. The field profiles are calculated numerically using COMSOL Multiphysics version 5.5a. The magnetic field is optimized between $\rho=\left[0, \rho_{c} / 4\right]$ and $z= \pm z^{\prime} / 2$; dashed red lines in (a),(b). Contours, in (a) only, show where the field deviates from the target field by $5 \%$ (dashed curves) and 1\% (dash-dot curves).

field. This can be seen from Fig. 3(d), which reveals small oscillations in the transverse field along the $z$ axis of the optimization region. To understand the difference between the fidelity of the field profiles generated by the two systems, we must analyze how the passive magnetic shield affects the fields from the planar current distributions, decomposing its response into zonal $(m=0)$ and tesseral $(m \neq 0)$ harmonic components. These harmonic responses relate to the variations in the induced pseudocurrent density, corresponding to the planar streamfunction (24), required to satisfy the boundary condition at the shield wall. Schematic approximations of the surface currents for the $m=0$ zonal and $m=1$ tesseral harmonic responses can be seen in Fig. 7. For the boundary condition on the cylindrical surface to be satisfied, the induced azimuthal pseudocurrents must mirror azimuthal current paths on the planar coil surfaces, so that the associated fields cancel in the region $\rho_{s}>\rho>\rho_{c}$. Since zonal responses are composed of simple circular loops, which can be formed in either a cylindrical or planar basis, the response of the magnetic shield enhances the magnetic field in the optimization region. Consequently, the shield amplifies the axial magnetic field by a factor of 2.39 at the shield's center, as shown in Figs. 5(b) and 5(c). The uniform field gradient therefore exhibits superior fidelity because the continuum response of the passive shield approximates a distributed cylindrical coil. The resulting system, composed of both the coil and shield, completely encloses the interior region, producing a high-fidelity magnetic field gradient.

The tesseral responses are more complicated, with the cylindrical surface of the magnetic shield acting to oppose the magnetic field generated by the planar system in the region $\rho<\rho_{s}$ due to the formation of saddle-type currents 

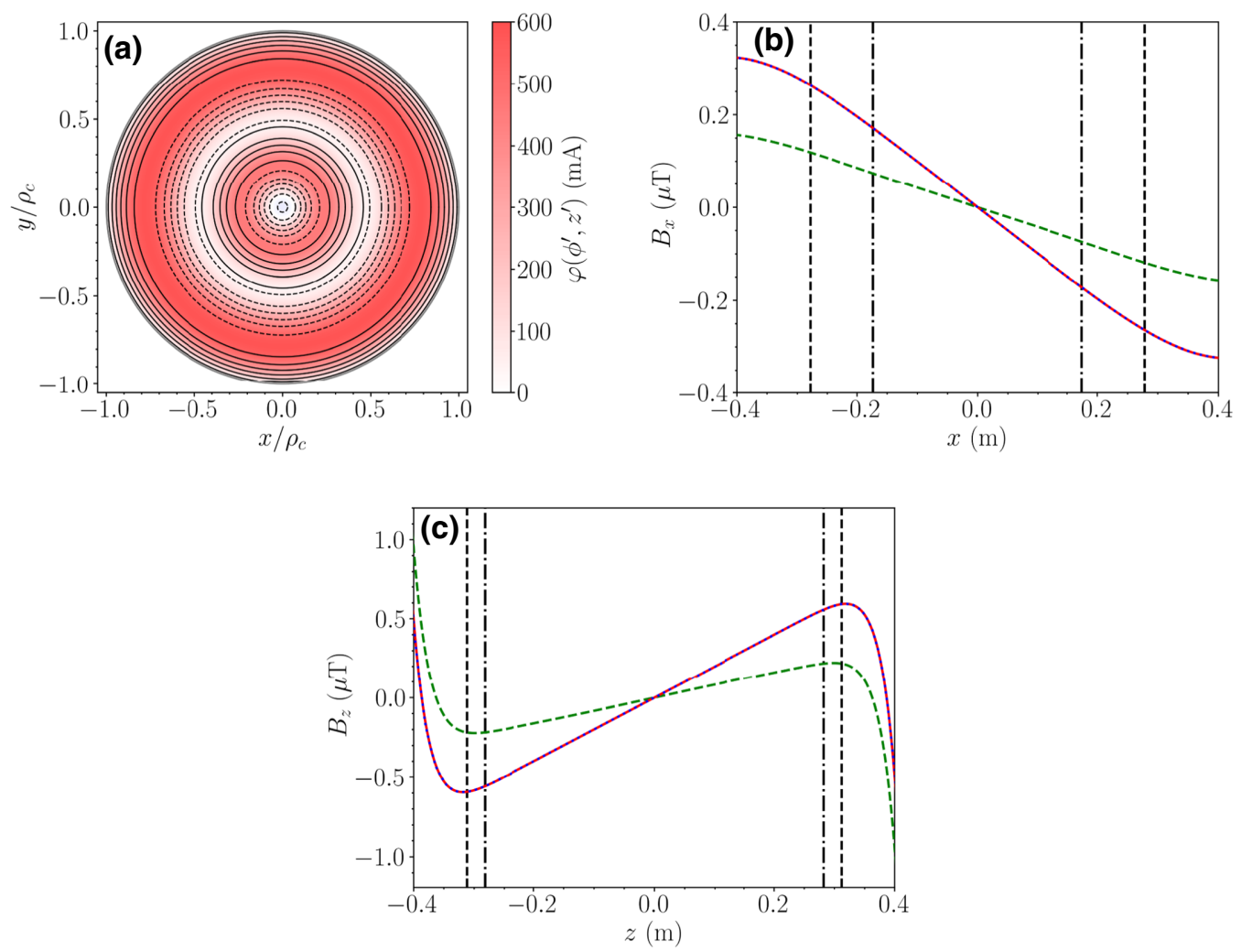

FIG. 5. Wire layouts (a) and performance (b),(c) of a hybrid active-passive system optimized to generate a linear variation of $B_{z}$ with $z$ position. Current flows on the surface of two disks of radius $\rho_{c}=0.45 \mathrm{~m}$, which are separated symmetrically from the origin and lie at $z^{\prime}= \pm 0.45 \mathrm{~m}$. The wire layouts are optimized to generate a linear axial field gradient, $d B_{z} / d z=2 \mu \mathrm{T} / \mathrm{m}$, along the $z$ axis of the cylinder through the harmonic $\mathbf{B}=(-x \hat{\mathbf{x}}-y \hat{\mathbf{y}}+2 z \hat{\mathbf{z}})$. The current-carrying planes are placed symmetrically inside a perfect closed magnetic shield of radius $\rho_{s}=0.5 \mathrm{~m}$ and length $L_{s}=1 \mathrm{~m}$ and the magnetic field is optimized between $\rho=\left[0, \rho_{c} / 4\right]$ and $z= \pm z^{\prime} / 2$, as shown in Fig. 2. The least squares optimization is performed with parameters $N=50, M=0, \beta=1.77 \times 10^{-9} \mathrm{~T}^{2} / \mathrm{W}, t=0.5$ $\mathrm{mm}$, and $\rho=1.68 \times 10^{-8} \Omega \mathrm{m}$. (a) Color map of the optimal current streamfunction calculated for the upper current-carrying plane in Fig. 2. Intensity of red shaded regions shows the streamfunction magnitude from low (white) to high (intense color). Solid and dashed black curves represent discrete wires with opposite senses of current flow, approximating the current continuum with $N_{\varphi}=12$ global contour levels. Streamfunction on the lower light gray plane in Fig. 2 is geometrically identical but the current direction is reversed. (b) Transverse magnetic field, $B_{x}$, calculated versus transverse position, $x$, for $y=z=0$, from the current continuum in (a) in three ways: analytically using Eqs. (26)-(28) (solid red curve); numerically using COMSOL Multiphysics version 5.5a, modeling the high-permeability cylinder as a perfect magnetic conductor (blue dotted curve); numerically without the high-permeability cylinder and using the Biot-Savart law with $N_{\varphi}=100$ contour levels (dashed green curve). Black lines enclose the regions where the calculated field deviates from the target field by $5 \%$ (dashed) and 1\% (dash-dot). (c) Axial magnetic field, $B_{z}$, calculated versus axial position, $z$, for $x=y=0$, from the current continuum in (a) in the same three ways as for (b).

[37]. These currents result from the required continuity of the induced azimuthal pseudocurrent density in a cylindrical basis. Because of the restricted current flow on the planar surface, the only way to mitigate the opposing field generated by the cylindrical surface of the shield is to minimize the magnetic field at the boundary, resulting in field coil designs that are oscillatory, as seen in Fig. 3(a). The response of the passive shield, for this configuration, reduces the magnetic field by a factor of 2.41 at the shield's center, as shown in Figs. 3(b) and 3(c).

The shield's response not only makes the optimization of tesseral harmonic fields more difficult in regions close to the cylindrical surface of the magnetic shield, but also has an effect on the level of fidelity that can be achieved over any region when the coils are in close proximity to the magnetic shield. To demonstrate this, we investigate the fidelity of the constant transverse field generated by optimized biplanar coils, similar to those in Fig. 3, within a magnetic shield whose radius varies over the range $\rho_{s}=$ $\left[\rho_{c}, 3 \rho_{c}\right]$. In Fig. 8(a) we plot field profiles at selected shield radii to show how the uniformity of the transverse field along the $x$ axis improves as the radius of the shield increases, as expected, due to the reduced inhomogeneities introduced by the cylindrical surface of the magnetic shield at distances further from the field coils. To evaluate the deviation in the field uniformity, we can calculate the mean 
(a)

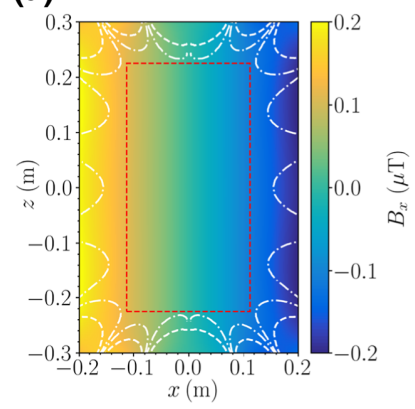

(b)

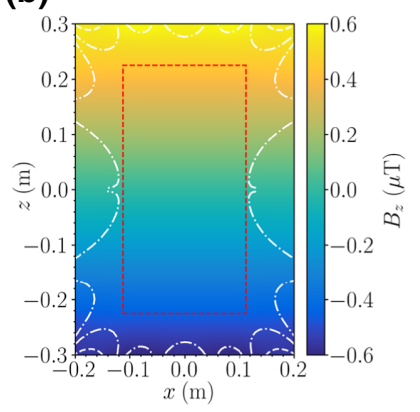

fields in Figs. 8(a) and 8(b) initially decreases rapidly as the radius of the magnetic shield increases, but approaches the wide shield limit when $\rho_{s} \sim 2 \rho_{c}$, at which point the field generated by the cylindrical surface of the magnetic shield becomes negligible. In the wide shield limit the predominant contribution from the magnetic shield can then be approximated through the use of the method of mirror images from two infinite parallel perfect magnetic conductors.

Although the pseudocurrents do not exist physically, analysis of the magnetic fields generated by the shield in response to the planar coils gives insight into how such coils should be designed in order to best realize a specified target field. For example, generating magnetic fields that require coil geometries with $m$-fold azimuthal symmetry causes the cylindrical surface of the magnetic shield to oppose the magnetic field generated by the planar coil. Consequently, if a tesseral field is required over a large radial region, the distance from this region to the planar coils and from the cylindrical shield surface should be minimized and maximized, respectively. The opposite is true for the fields generated by the planar end caps of the shield. When the biplanar coils are located near the end caps, where $-L_{s} / 2<-z^{\prime}<z<z^{\prime}<L_{s} / 2$, but radially distant from the cylindrical surface of the magnetic shield, the reflected pseudocurrents generated by the method of mirror

rms error, which represents the averaged deviation of the transverse field from perfect uniformity. In Fig. 8(b), we evaluate the mean rms error in the transverse field along the $z$ axis of the optimization region as the radius of the magnetic shield increases. The uniformity of the optimized

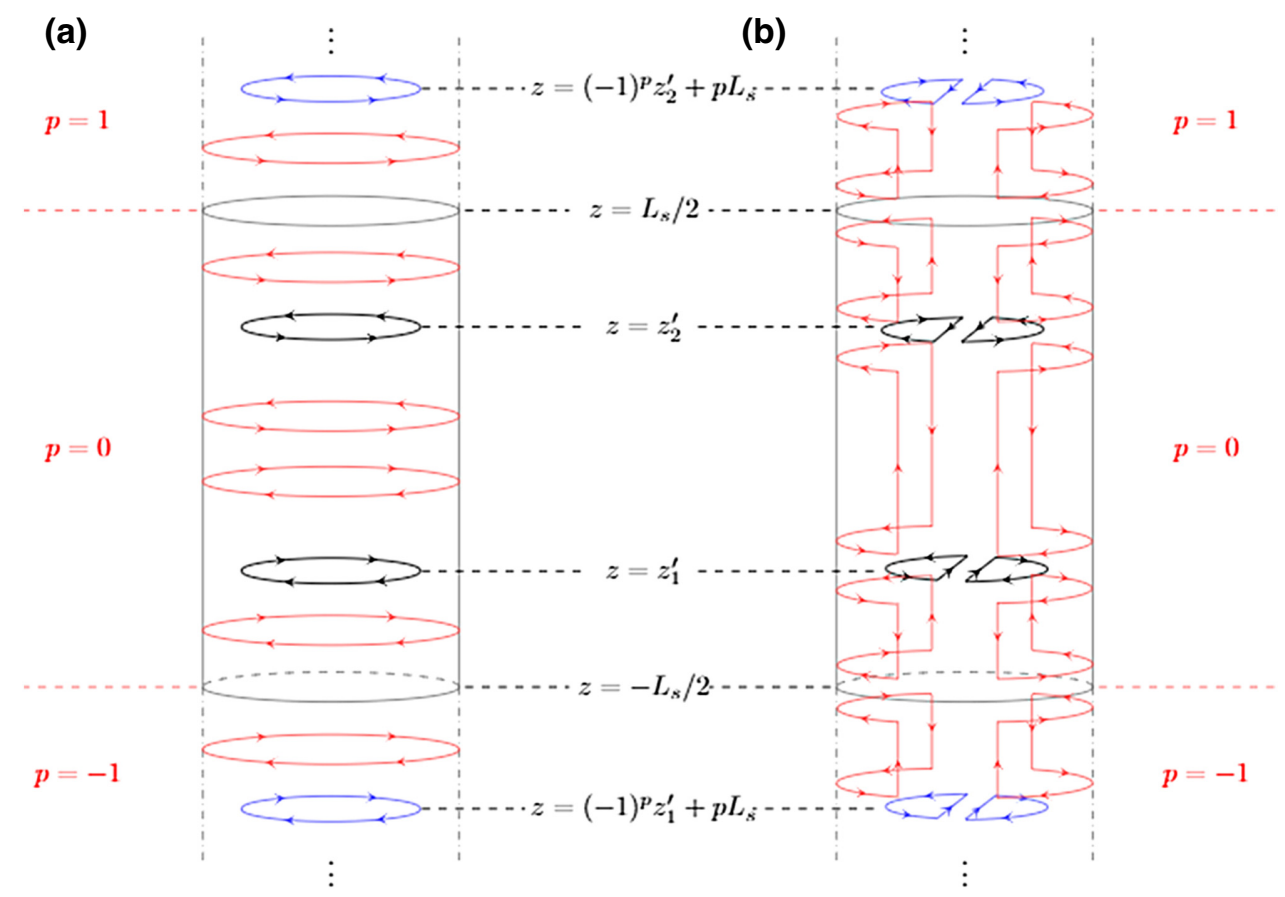

FIG. 7. Schematic diagram showing how the approximate zonal (a) and tesseral (b) harmonic responses are generated by simple current loops with cylindrical and $m$-fold azimuthal symmetries, respectively. The discrete current loops (black) are located at $z=z_{1}^{\prime}$ and $z=z_{2}^{\prime}$ with their $p$ th reflected pseudoimage currents (red) generated by the surface of the cylindrical shield at $\rho=\rho_{s}$ and by the planar end caps at $z= \pm L_{s} / 2$, in accordance with the method of images described by Eq. (24). Images of the two planar (black) coils resulting from the end caps are located at $z=(-1)^{p} z_{1}^{\prime}+p L_{s}$ and $z=(-1)^{p} z_{2}^{\prime}+p L_{s}$, respectively, where $p \in \mathbb{Z}$ (two such image coils are shown blue). For all odd reflections, the axial current direction is reversed. 

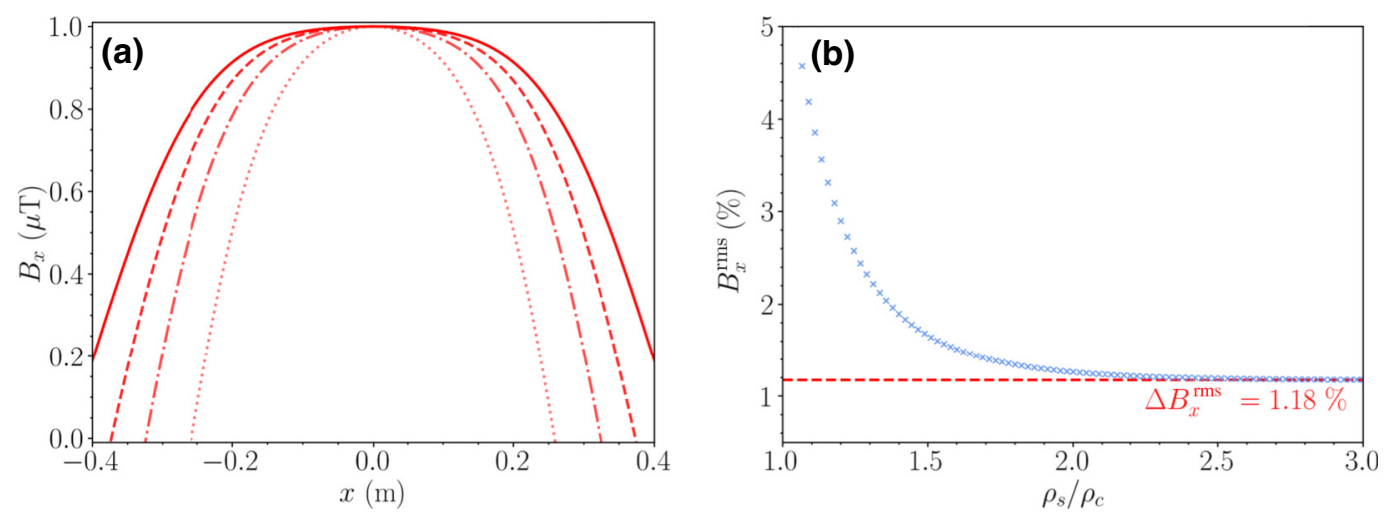

FIG. 8. Improvement in the performance of a hybrid active-passive system, optimized to generate a constant transverse field $B_{0}=1 \mu \mathrm{T}$, upon increasing the radius of the passive shield. Current flows on the surface of two disks of radius $\rho_{c}=0.45 \mathrm{~m}$, which are separated symmetrically from the origin and lie at $z^{\prime}= \pm 0.45 \mathrm{~m}$. The wire layouts are optimized to generate constant $B_{0}=1 \mu \mathrm{T}$ between $\rho=\left[0, \rho_{c} / 4\right]$ and $z= \pm z^{\prime} / 2$, as shown in Fig. 2. The current-carrying planes lie within the bore of a perfect closed magnetic shield of radius $\rho_{s}=\left[\rho_{c}, 3 \rho_{c}\right]$ and length $L_{s}=1 \mathrm{~m}$. Each least squares optimization is performed with parameters $N=50, M=1$, $\beta=1.77 \times 10^{-9} \mathrm{~T}^{2} / \mathrm{W}, t=0.5 \mathrm{~mm}$, and $\rho=1.68 \times 10^{-8} \Omega \mathrm{m}$. (a) Transverse magnetic field, $B_{x}$, calculated analytically versus transverse position, $x$, using Eqs. (26)-(28) (red curves), where $\rho_{s} / \rho_{c}=1.01$ (dotted curve), $\rho_{s} / \rho_{c}=1.25$ (dash-dot curve), $\rho_{s} / \rho_{c}=1.5$ (dashed curve), and $\rho_{s} / \rho_{c}=2.5$ (solid curve). (b) Root mean square (rms) deviation, $\Delta B_{x}^{\text {rms }}$, between the calculated and target fields evaluated along the axis of the optimized field region and plotted as a function of $\rho_{s} / \rho_{c}$. Light blue crosses show $\Delta B_{x}^{\text {rms }}$ values calculated analytically using Eqs. (26)-(28). Horizontal dashed red line shows the analytical value of $\Delta B_{x}^{\text {rms }}=1.18 \%$ obtained in the wide shield limit $\left(\rho_{s} / \rho_{c} \gg 1\right)$.

images provide a field similar to that of the planar coils, resulting in a field that is magnified. Consequently, biplanar coils are desirable in magnetic shields with large radii and small aspect ratios, $L_{s} /\left(2 \rho_{s}\right)<0.5$. In comparison, the magnetic field generated by the shield in response to coils contained on a cylindrical surface [33] shows the opposite effect. Tesseral fields generated by cylindrical coils are enhanced by the cylindrical surface and are reduced by the planar end caps, favoring long magnetic shields with $L_{s} /\left(2 \rho_{s}\right)>0.5$. Because of the conflicting conditions on the geometries (i.e., planar or cylindrical) of the coils and the magnetic shield, a system composed of coupled planar and cylindrical coils may have advantages for generating desired tesseral field profiles with the greatest accuracy.

Intrinsic inaccuracies in calculating the optimum current density and approximating the current continuum by discrete wires are not the only sources of error that must be considered when designing fields using our method. Particularly for the tesseral harmonic field generating coils, wire patterns may be highly meandering, making them hard to manufacture and, due to their high resistance, power consuming. When manufacturing these systems, the achievable field fidelity is limited by the discretization of the continuum current flow pattern and by the creation of magnetic dipoles via the interaction between separate current streamlines and the wires that follow them. Fortunately, new ways to realize the current continuum are emerging due to advances in foldable printed circuit boards [39] and three-dimensional printing technologies [40], which enable more complex coils to be made accurately. It should also be noted that approximating a perfect magnetic shield by a material of finite permeability $\mu_{r}=20000$ and thickness $d=1 \mathrm{~mm}$ only introduces small deviations in the model of $0.005 \%$ [33], which is much less than the error introduced in the desired field by the coil designs. As a result, given an accurate representation of the current continuum, our methodology can be used reliably to generate target magnetic fields in high-permeability environments. The PYTHON code used to design arbitrary the planar coils in a magnetically shielded cylinder is openly available from GitHub and can be cited at Ref. [38]. Verification using COMSOL Multiphysics requires a valid license.

\section{CONCLUSION}

Here we formulate an analytical model to calculate the magnetic field generated by an arbitrary static current distribution confined to a plane whose normal is parallel to the axis of a finite closed high-permeability cylinder. Our formalism is based on a Green's function expansion of the vector potential generated by a planar coil system. To satisfy the boundary conditions at the surface of the cylinder, we assume that it is a perfect magnetic conductor and express the induced magnetization in terms of a pseudocurrent density. Because of the shared azimuthal symmetries of the planar current flows and the induced pseudocurrent on the cylindrical surface of the magnetic shield, the response of the magnetic shield can be expressed in terms of the planar current distribution. We use this formalism to enable a priori optimization of the planar current 
distribution required to generate specified target magnetic field profiles by combining a Fourier-Bessel decomposition of the current flow with a quadratic minimization procedure.

We demonstrate this optimization methodology by using it to design biplanar coils that accurately generate a constant transverse field, $B_{x}$, across the cylinder, and a linear gradient field, $d B_{z} / d z$, along the axis of the cylinder. Predictions from our analytical model agree well with subsequent forward finite element simulations, validating our methodology. We quantify the interaction of planar systems with a closed finite high-permeability cylinder and find that all coil designs without complete azimuthal symmetry induce magnetic fields in the cylindrical surface of the shield that opposed the field generated by the planar current source. Conversely, planar end caps have the opposite effect and amplify the field from the planar current source.

Our analytical model and optimization procedure extends the range of current geometries that can be tailored within finite closed cylindrical magnetic shields in order to accurately generate specified target field profiles. This enables the development of systems that require the best magnetic field control that can be achieved subject to size, weight, power, and cost constraints. It may also enable hybrid active-passive planar coils to be retrofitted to existing cylindrical magnetic shields in order to improve their performance for a low cost and without disrupting existing experimental setups. In the future, combining planar and cylindrical coils could enable ultraprecise magnetic fields to be generated through even larger interior fractions of magnetic shields.

\section{ACKNOWLEDGMENTS}

We acknowledge support from the UK Quantum Technology Hub Sensors and Timing, funded by the Engineering and Physical Sciences Research Council (EP/M013294/1). M.P., P.J.H., T.M.F., M.J.B., and R.B. declare that they have a patent pending to the UK Government Intellectual Property Office (Application No. 1913549.0) regarding the magnetic field optimization techniques described in this work. N.H., M.J.B., and R.B. also declare financial interests in a University of Nottingham spin-out company, Cerca. The authors have no other competing financial interests.

\section{APPENDIX A: MAGNETIC FIELD DERIVATION}

In order to calculate the magnetic field, we must first determine both the Fourier transform of the streamfunction and the induced Fourier pseudocurrent density. Substituting Eq. (25) into Eq. (12) and integrating over the azimuthal angle yields

$$
\varphi^{m}(k)=\frac{\rho_{c}}{2} \sum_{n=1}^{N} \sum_{m^{\prime}=0}^{M}\left[W_{n m^{\prime}}\left(\delta_{m m^{\prime}}+\delta_{m,-m^{\prime}}\right)-i Q_{n m^{\prime}}\left(\delta_{m m^{\prime}}-\delta_{m,-m^{\prime}}\right)\right] \int_{0}^{\rho_{c}} d \rho^{\prime} \rho^{\prime} J_{m}\left(k \rho^{\prime}\right) J_{m^{\prime}}\left(\frac{\rho_{n m^{\prime}} \rho^{\prime}}{\rho_{c}}\right) .
$$

Upon substituting Eq. (A1) into the expressions for the magnetic field, Eqs. (20)-(22), the $\delta$ functions collapse the infinite summation, resulting in every Bessel function of order $m$ being replaced by one of order $m^{\prime}$. Hence, without loss of generality, applying a Bessel function product identity (Eq. 5.54.1 in Ref. [41]), we may now write

$$
\varphi^{m}(k)=\frac{\rho_{c}^{3}}{2} \sum_{n=1}^{N} \sum_{m^{\prime}=0}^{M}\left[W_{n m^{\prime}}\left(\delta_{m m^{\prime}}+\delta_{m,-m^{\prime}}\right)-i Q_{n m^{\prime}}\left(\delta_{m m^{\prime}}-\delta_{m,-m^{\prime}}\right)\right] \frac{\rho_{n m^{\prime}}}{k^{2} \rho_{c}^{2}-\rho_{n m^{\prime}}^{2}} J_{m^{\prime}}\left(k \rho_{c}\right) J_{m^{\prime}}^{\prime}\left(\rho_{n m^{\prime}}\right) .
$$

Similarly, we may also calculate the induced Fourier pseudocurrent density. Substituting Eq. (25) into Eq. (24) and integrating over the azimuthal coordinate yields

$$
\begin{aligned}
J_{\phi}^{m p}(k)= & \frac{\rho_{c} e^{-i k\left[(-1)^{p} z^{\prime}+p L_{s}\right]}}{2 \rho_{s}|k| I_{m}\left(|k| \rho_{s}\right) K_{m}^{\prime}\left(|k| \rho_{s}\right)} \sum_{n=1}^{N} \sum_{m^{\prime}=0}^{M}\left[W_{n m^{\prime}}\left(\delta_{m m^{\prime}}+\delta_{m,-m^{\prime}}\right)-i Q_{n m^{\prime}}\left(\delta_{m m^{\prime}}-\delta_{m,-m^{\prime}}\right)\right] \\
& \times \int_{0}^{\infty} d \tilde{k} \frac{\tilde{k}^{3}}{\tilde{k}^{2}+k^{2}} J_{m}\left(\tilde{k} \rho_{s}\right) \int_{0}^{\rho_{c}} d \rho^{\prime} \rho^{\prime} J_{m^{\prime}}\left(\tilde{k} \rho^{\prime}\right) J_{m^{\prime}}\left(\frac{\rho_{n m^{\prime}} \rho^{\prime}}{\rho_{c}}\right) .
\end{aligned}
$$

To simplify these expressions, we evaluate the integral over $\tilde{k}$ first and then separate the integrand using partial fractions to yield

$$
\int_{0}^{\infty} d \tilde{k} \frac{\tilde{k}^{3}}{\tilde{k}^{2}+k^{2}} J_{m}\left(\tilde{k} \rho_{s}\right) J_{m}\left(\tilde{k} \rho^{\prime}\right)=\int_{0}^{\infty} d \tilde{k} \tilde{k} J_{m}\left(\tilde{k} \rho_{s}\right) J_{m}\left(\tilde{k} \rho^{\prime}\right)-k^{2} \int_{0}^{\infty} d \tilde{k} \frac{\tilde{k}}{\tilde{k}^{2}+k^{2}} J_{m}\left(\tilde{k} \rho_{s}\right) J_{m}\left(\tilde{k} \rho^{\prime}\right) .
$$


Inspecting Eq. (A4) we see that the first component is simply the orthogonality relation between Bessel functions, and the second has a known solution (Eq. 6.541.1 in [41]). Hence, we can state that

$$
\int_{0}^{\infty} d \tilde{k} \frac{\tilde{k}^{3}}{\tilde{k}^{2}+k^{2}} J_{m}\left(\tilde{k} \rho_{s}\right) J_{m}\left(\tilde{k} \rho^{\prime}\right)=\frac{\delta\left(\rho^{\prime}-\rho_{s}\right)}{\rho^{\prime}}-k^{2} I_{m^{\prime}}\left(|k| \rho^{\prime}\right) K_{m^{\prime}}\left(|k| \rho_{s}\right) .
$$

However, since $\rho^{\prime}<\rho_{s}$, the first term in Eq. (A5) can be neglected. Inserting Eq. (A5) into Eq. (A3) and integrating over $\rho^{\prime}$ results in the expression

$$
\begin{aligned}
J_{\phi}^{m p}(k)= & \frac{\rho_{c} k^{2} e^{-i k\left[(-1)^{p} z^{\prime}+p L_{s}\right]}}{2 \rho_{s}|k| I_{m}\left(|k| \rho_{s}\right) K_{m}^{\prime}\left(|k| \rho_{s}\right)} \sum_{n=1}^{N} \sum_{m^{\prime}=0}^{M}\left[W_{n m^{\prime}}\left(\delta_{m m^{\prime}}+\delta_{m,-m^{\prime}}\right)-i Q_{n m^{\prime}}\left(\delta_{m m^{\prime}}-\delta_{m,-m^{\prime}}\right)\right] \\
& \times \frac{\rho_{n m^{\prime}}}{k^{2} \rho_{c}^{2}+\rho_{n m^{\prime}}^{2}} J_{m^{\prime}}^{\prime}\left(\rho_{n m^{\prime}}\right) I_{m^{\prime}}\left(|k| \rho_{c}\right) K_{m^{\prime}}\left(|k| \rho_{s}\right) .
\end{aligned}
$$

Substituting Eqs. (A2) and (A6) into Eqs. (20)-(22), we can express the summation over the exponentials associated with the infinite reflections of the planar streamfunction as

$$
\sum_{p=-\infty}^{\infty} e^{-k\left|z-(-1)^{p} z^{\prime}+p L_{S}\right|}=e^{-k\left|z-z^{\prime}\right|}+\frac{2}{e^{2 k L}-1}\left\{e^{k L} \cosh \left[k\left(z+z^{\prime}\right)\right]+\cosh \left[k\left(z-z^{\prime}\right)\right]\right\}
$$

and

$$
\begin{aligned}
& \sum_{p=-\infty}^{\infty} \frac{z-(-1)^{p} z^{\prime}+p L_{s}}{\left|z-(-1)^{p} z^{\prime}+p L_{s}\right|} e^{-k\left|z-(-1)^{p} z^{\prime}+p L_{s}\right|} \\
& \quad=\frac{z-z^{\prime}}{\left|z-z^{\prime}\right|} e^{-k\left|z-z^{\prime}\right|}-\frac{2}{e^{2 k L}-1}\left\{e^{k L} \sinh \left[k\left(z+z^{\prime}\right)\right]+\sinh \left[k\left(z-z^{\prime}\right)\right]\right\}
\end{aligned}
$$

Similarly, the summation over the infinite pseudocurrent reflections can be written as a Fourier series expansion,

$$
\sum_{p=-\infty}^{\infty} e^{i k\left[z-(-1)^{p} z^{\prime}+p L_{S}\right]}=\frac{\pi}{L} \sum_{p=-\infty}^{\infty} \delta\left(k-\frac{p \pi}{L}\right)\left(e^{i k\left(z+z^{\prime}-L\right)}+e^{i k\left(z-z^{\prime}\right)}\right) .
$$

Using these expansions, we may finally write

$$
\begin{aligned}
& B_{\rho}(\rho, \phi, z)=\frac{\mu_{0} \rho_{c}^{3}}{2} \sum_{n=1}^{N} \sum_{m=0}^{M} \rho_{n m} J_{m}^{\prime}\left(\rho_{n m}\right)\left[W_{n m} \cos (m \phi)+Q_{n m} \sin (m \phi)\right] B_{\rho}^{n m}(\rho, z), \\
& B_{\phi}(\rho, \phi, z)=\frac{\mu_{0} \rho_{c}^{3}}{2 \rho} \sum_{n=1}^{N} \sum_{m=0}^{M} m \rho_{n m} J_{m}^{\prime}\left(\rho_{n m}\right)\left[W_{n m} \sin (m \phi)-Q_{n m} \cos (m \phi)\right] B_{\phi}^{n m}(\rho, z), \\
& B_{z}(\rho, \phi, z)=\frac{\mu_{0} \rho_{c}^{3}}{2} \sum_{n=1}^{N} \sum_{m=0}^{M} \rho_{n m} J_{m}^{\prime}\left(\rho_{n m}\right)\left[W_{n m} \cos (m \phi)+Q_{n m} \sin (m \phi)\right] B_{z}^{n m}(\rho, z),
\end{aligned}
$$

where

$$
B_{\rho}^{n m}(\rho, z)=-\int_{0}^{\infty} d k k^{2} \sigma\left(k ; z, z^{\prime}, L_{s}\right) \frac{J_{m}^{\prime}(k \rho) J_{m}\left(k \rho_{c}\right)}{k^{2} \rho_{c}^{2}-\rho_{n m}^{2}}-\sum_{p=1}^{\infty} \tilde{p}|\tilde{p}| \lambda_{p}\left(z, z^{\prime}, L_{s}\right) \frac{I_{m}^{\prime}(|\tilde{p}| \rho) I_{m}\left(|\tilde{p}| \rho_{c}\right) K_{m}\left(|\tilde{p}| \rho_{s}\right)}{I_{m}\left(|\tilde{p}| \rho_{s}\right)\left(|\tilde{p}|^{2} \rho_{c}^{2}+\rho_{n m}^{2}\right)}
$$




$$
\begin{aligned}
& B_{\phi}^{n m}(\rho, z)=\int_{0}^{\infty} d k k \sigma\left(k ; z, z^{\prime}, L_{s}\right) \frac{J_{m}(k \rho) J_{m}\left(k \rho_{c}\right)}{k^{2} \rho_{c}^{2}-\rho_{n m}^{2}}+\sum_{p=1}^{\infty} \tilde{p} \lambda_{p}\left(z, z^{\prime}, L_{s}\right) \frac{I_{m}(|\tilde{p}| \rho) I_{m}\left(|\tilde{p}| \rho_{c}\right) K_{m}\left(|\tilde{p}| \rho_{s}\right)}{I_{m}\left(|\tilde{p}| \rho_{s}\right)\left(|\tilde{p}|^{2} \rho_{c}^{2}+\rho_{n m}^{2}\right)} \\
& B_{z}^{n m}(\rho, z)=\int_{0}^{\infty} d k k^{2} \gamma\left(k ; z, z^{\prime}, L_{s}\right) \frac{J_{m}(k \rho) J_{m}\left(k \rho_{c}\right)}{k^{2} \rho_{c}^{2}-\rho_{n m}^{2}}-\sum_{p=1}^{\infty} \tilde{p}^{2} \tau_{p}\left(z, z^{\prime}, L_{s}\right) \frac{I_{m}(|\tilde{p}| \rho) I_{m}\left(|\tilde{p}| \rho_{c}\right) K_{m}\left(|\tilde{p}| \rho_{s}\right)}{I_{m}\left(|\tilde{p}| \rho_{s}\right)\left(|\tilde{p}|^{2} \rho_{c}^{2}+\rho_{n m}^{2}\right)}
\end{aligned}
$$

where

$$
\begin{aligned}
& \gamma\left(k ; z, z^{\prime}, L_{s}\right)=e^{-k\left|z-z^{\prime}\right|}+\frac{2}{e^{2 k L_{s}}-1}\left\{e^{k L_{s}} \cosh \left[k\left(z+z^{\prime}\right)\right]+\cosh \left[k\left(z-z^{\prime}\right)\right]\right\}, \\
& \sigma\left(k ; z, z^{\prime}, L_{s}\right)=\frac{z-z^{\prime}}{\left|z-z^{\prime}\right|} e^{-k\left|z-z^{\prime}\right|}-\frac{2}{e^{2 k L_{s}}-1}\left\{e^{k L_{s}} \sinh \left[k\left(z+z^{\prime}\right)\right]+\sinh \left[k\left(z-z^{\prime}\right)\right]\right\}, \\
& \lambda_{p}\left(z, z^{\prime}, L_{s}\right)=\frac{2}{L_{s}}\left\{(-1)^{p} \sin \left[\tilde{p}\left(z+z^{\prime}\right)\right]+\sin \left[\tilde{p}\left(z-z^{\prime}\right)\right]\right\}, \\
& \tau_{p}\left(z, z^{\prime}, L_{s}\right)=\frac{2}{L_{s}}\left\{(-1)^{p} \cos \left[\tilde{p}\left(z+z^{\prime}\right)\right]+\cos \left[\tilde{p}\left(z-z^{\prime}\right)\right]\right\},
\end{aligned}
$$

and $\tilde{p}=p \pi / L_{s}$.

\section{APPENDIX B: POWER DISSIPATION}

The power dissipated in the surface of a circular planar current source of thickness $t$ and resistivity $\varrho$ is given by

$$
P=\frac{\varrho}{t} \int_{0}^{\rho_{c}} d \rho^{\prime} \rho^{\prime} \int_{0}^{2 \pi} d \phi^{\prime}\left|J_{\rho}\left(\rho^{\prime}, \phi^{\prime}\right)\right|^{2}+\left|J_{\phi}\left(\rho^{\prime}, \phi^{\prime}\right)\right|^{2} .
$$

We can substitute the streamfunction (25) into the continuity relations (7) to obtain

$$
\begin{aligned}
& J_{\rho}\left(\rho^{\prime}, \phi^{\prime}\right)=\left[H\left(\rho^{\prime}-\rho_{c}\right)-H\left(\rho^{\prime}\right)\right] \frac{\rho_{c}}{\rho^{\prime}} \sum_{n=1}^{N} \sum_{m=0}^{M} m J_{m}\left(\frac{\rho_{n m} \rho^{\prime}}{\rho_{c}}\right)\left[W_{n m} \sin \left(m \phi^{\prime}\right)-Q_{n m} \cos \left(m \phi^{\prime}\right)\right], \\
& J_{\phi}\left(\rho^{\prime}, \phi^{\prime}\right)=\left[H\left(\rho^{\prime}-\rho_{c}\right)-H\left(\rho^{\prime}\right)\right] \sum_{n=1}^{N} \sum_{m=0}^{M} \rho_{n m} J_{m}^{\prime}\left(\frac{\rho_{n m} \rho^{\prime}}{\rho_{c}}\right)\left[W_{n m} \cos \left(m \phi^{\prime}\right)+Q_{n m} \sin \left(m \phi^{\prime}\right)\right] .
\end{aligned}
$$

Inserting Eqs. (B2) and (B3) into Eq. (B1) and integrating over the azimuthal component results in

$$
\begin{aligned}
P= & \frac{\varrho}{t} \pi \rho_{c}^{2} \sum_{n=1}^{N} \sum_{m=0}^{M}\left[\left(1+\delta_{m 0}\right) W_{n m}^{2}+\left(1-\delta_{m 0}\right) Q_{n m}^{2}\right] \\
& \times \int_{0}^{1} d \tilde{\rho}\left[\frac{2 m^{2}}{\tilde{\rho}} J_{m}\left(\rho_{n m} \tilde{\rho}\right)^{2}-\rho_{n m}^{2} \tilde{\rho} J_{m-1}\left(\rho_{n m} \tilde{\rho}\right) J_{m+1}\left(\rho_{n m} \tilde{\rho}\right)\right],
\end{aligned}
$$

which, when integrated over the radial component, can be expressed as

$$
P=\frac{\varrho}{t} \pi \rho_{c}^{2} \sum_{n=1}^{N} W_{n 0}^{2} \rho_{n 0}^{2} J_{1}\left(\rho_{n 0}\right)^{2}
$$

for $m=0$, and

$$
\begin{aligned}
P= & \frac{\varrho}{t} \pi \rho_{c}^{2} \sum_{n=1}^{N} \sum_{m=1}^{M}\left(W_{n m}^{2}+Q_{n m}^{2}\right)\left(\frac{\rho_{n m}}{2}\right)^{2 m} \frac{1}{m !(m-1) !}\left[{ }_{2} \tilde{F}_{3}\left(m, m+\frac{1}{2} ; m+1, m+1,2 m+1 ;-\rho_{n m}^{2}\right)\right. \\
& \left.-\frac{\rho_{n m}^{2}}{2(m+1)^{2}}{ }_{3} \tilde{F}_{4}\left(m+\frac{1}{2}, m+1, m+1 ; m, m+2, m+2,2 m+1 ;-\rho_{n m}^{2}\right)\right]
\end{aligned}
$$


for $m \in \mathbb{Z}^{+}$, where ${ }_{i} \tilde{F}_{j}$ is the regularized hypergeometric function. Some specific evaluations of Eq. (B6) are

$$
\begin{gathered}
P=\frac{\varrho}{2 t} \pi \rho_{c}^{2} \sum_{n=1}^{N}\left(W_{n 1}^{2}+Q_{n 1}^{2}\right) \rho_{n 1}^{2} J_{0}\left(\rho_{n 1}\right)^{2} \quad \text { for } m=1, \\
P=\frac{\varrho}{2 t} \pi \rho_{c}^{2} \sum_{n=1}^{N}\left(W_{n 2}^{2}+Q_{n 2}^{2}\right)\left[\left(\rho_{n 2}^{2}-4\right) J_{0}\left(\rho_{n 2}\right)^{2}-\frac{2\left(\rho_{n 2}^{2}-8\right)}{\rho_{n 2}} J_{0}\left(\rho_{n 2}\right) J_{1}\left(\rho_{n 2}\right)+\frac{\rho_{n 2}^{4}-16}{\rho_{n 2}^{2}} J_{1}\left(\rho_{n 2}\right)^{2}\right] \quad \text { for } m=2, \quad \text { (B8) } \\
P=\frac{\varrho}{2 t} \pi \rho_{c}^{2} \sum_{n=1}^{N}\left(W_{n 3}^{2}+Q_{n 3}^{2}\right)\left[\frac{\rho_{n 3}^{4}-96}{\rho_{n 3}^{2}} J_{0}\left(\rho_{n 3}\right)^{2}-48\left(\rho_{n 3}^{2}-8\right) \rho_{n 3}^{3} J_{0}\left(\rho_{n 3}\right) J_{1}\left(\rho_{n 3}\right)+\frac{\rho_{n 3}^{6}-10 \rho_{n 3}^{4}+96 \rho_{n 3}^{2}-384}{\rho_{n 3}^{4}} J_{1}\left(\rho_{n 3}\right)^{2}\right] \\
\text { for } m=3 .
\end{gathered}
$$

\section{APPENDIX C: EXAMPLE COIL DESIGNS}

To further validate our design process, in Figs. 9 and 10 we show additional biplanar hybrid active-passive systems and analyze their behavior. These systems are designed using our open-access PYTHON code [38]. The coordinate axes and magnetic field plots are labeled in the same way as the systems presented in the main text. For both systems, the coils lie in the $z^{\prime}= \pm 0.45 \mathrm{~m}$ planes, as in the main text and shown in Fig. 2, and the fields are optimized along the $z$ axis between $z= \pm z^{\prime} / 2$. The design in Fig. 9 generates a linear transverse field gradient, $d B_{x} / d z$, whose spatial variation matches the harmonic $\mathbf{B}=(z \hat{\mathbf{x}}+x \hat{\mathbf{z}})$ between two
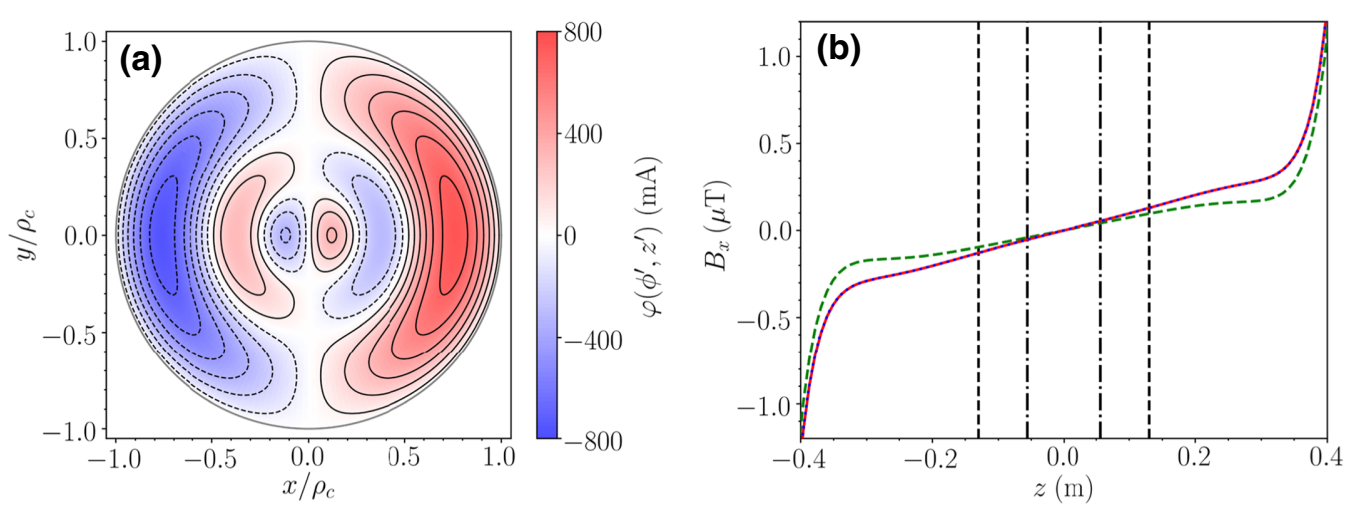

FIG. 9. Wire layouts (a) and performance (b) of a hybrid active-passive system optimized to generate a linear variation of $B_{x}$ with $z$ position. Current flows on the surface of two disks of radius $\rho_{c}=0.45 \mathrm{~m}$, which are separated symmetrically from the origin and lie at $z^{\prime}= \pm 0.45 \mathrm{~m}$. The wire layouts are optimized to generate a linear transverse field gradient, $d B_{x} / d z=1 \mu \mathrm{T} / \mathrm{m}$, along the $z$ axis of the cylinder through the harmonic field profile $\mathbf{B}=(z \hat{\mathbf{x}}+x \hat{\mathbf{z}})$. The current-carrying planes are placed symmetrically inside a perfect closed magnetic shield of radius $\rho_{s}=0.5 \mathrm{~m}$ and length $L_{s}=1 \mathrm{~m}$ and the magnetic field is optimized along the $z$ axis between $z= \pm z^{\prime} / 2$. The least squares optimization is performed with parameters $N=100, M=1, \beta=1.77 \times 10^{-8} \mathrm{~T}^{2} / \mathrm{W}, t=0.5 \mathrm{~mm}$, and $\rho=1.68 \times 10^{-8} \Omega \mathrm{m}$. (a) Color map of the optimal current streamfunction calculated for the upper current-carrying plane in Fig. 2 [blue and red shaded regions correspond to current counterflows and their intensity shows the streamfunction magnitude from low (white) to high (intense color)]. Solid and dashed black curves represent discrete wires with opposite senses of current flow, approximating the current continuum with $N_{\varphi}=12$ global contour levels. Streamfunction on the lower light brown plane in Fig. 2 is geometrically identical but the current direction is reversed. (b) Transverse magnetic field, $B_{x}$, calculated versus axial position, $z$, from the current continuum in (a) in three ways: analytically using Eqs. (26)-(28) (solid red curve); numerically using COMSOL Multiphysics version 5.5a, modeling the high-permeability cylinder as a perfect magnetic conductor (blue dotted curve); numerically without the high-permeability cylinder and using the Biot-Savart law with $N_{\varphi}=100$ contour levels (dashed green curve). Black lines enclose the regions where the axial field gradient deviates by $5 \%$ (dashed) and $1 \%$ (dash-dot). 

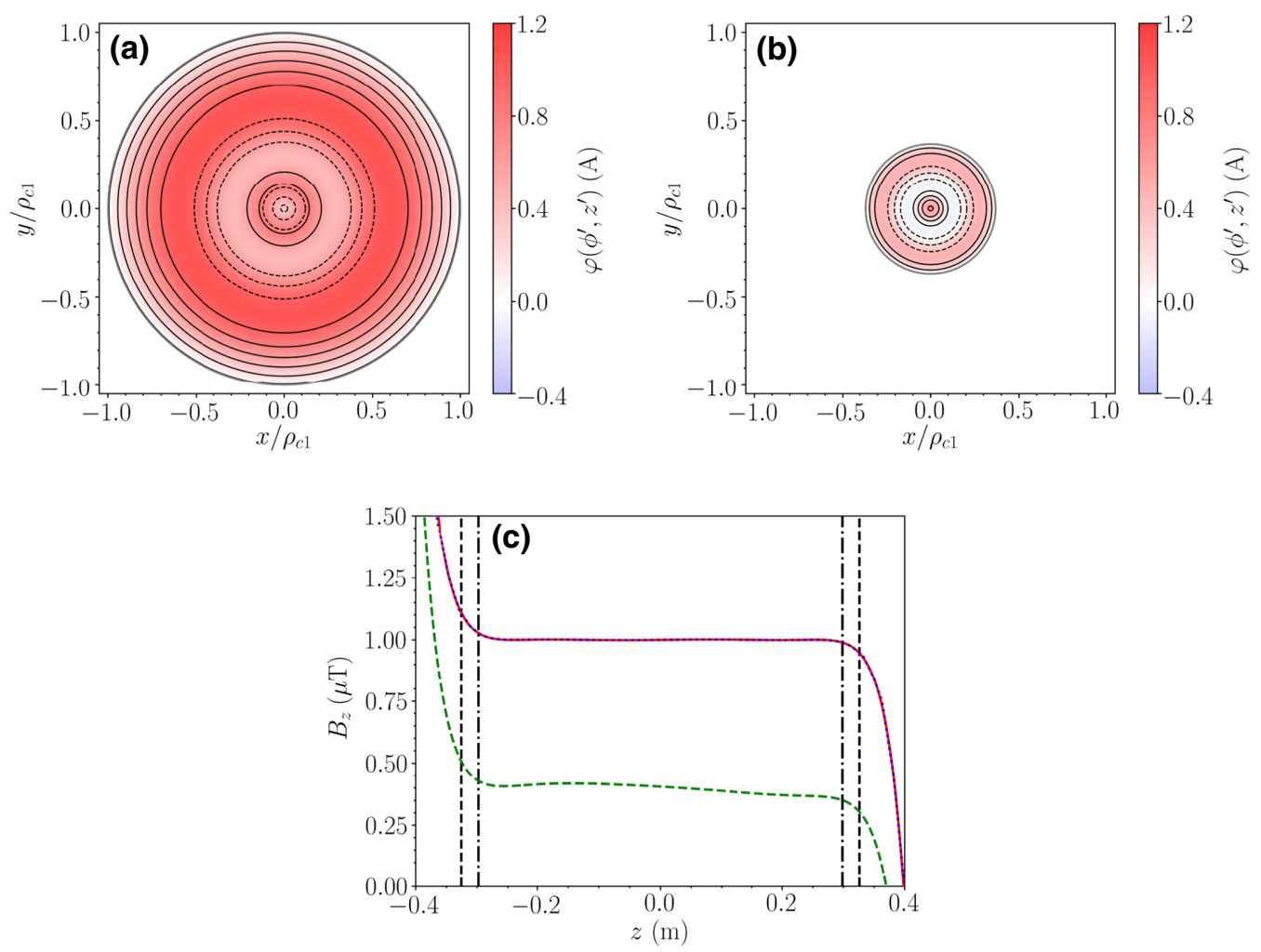

FIG. 10. Wire layouts (a),(b) and performance (c) of a hybrid active-passive linear system optimized to generate a constant axial field, $B_{z}$. Current flows on the surface of two disks of radii $\rho_{c 1}=0.95 \mathrm{~m}$ (on the upper current-carrying plane in Fig. 2) and $\rho_{c 2}=0.35 \mathrm{~m}$ (on the lower current-carrying plane in Fig. 2), respectively, which are separated symmetrically from the origin and lie at $z^{\prime}= \pm 0.45 \mathrm{~m}$. The wire layouts are optimized to generate a constant axial field, $B_{0}=1 \mu \mathrm{T}$, along the cylinder and parallel to its axis of symmetry. The current-carrying planes are placed symmetrically inside a perfect closed magnetic shield of radius $\rho_{s}=1 \mathrm{~m}$ and length $L_{s}=1 \mathrm{~m}$ and the magnetic field is optimized along the $z$ axis between $z= \pm z^{\prime} / 2$. The least squares optimization is performed with parameters $N=100, M=0, \beta=1.77 \times 10^{-8}, T^{2} / W, t=0.5 \mathrm{~mm}$, and $\rho=1.68 \times 10^{-8} \Omega \mathrm{m}$. (a),(b) Color maps of the optimal current streamfunction on the upper and lower planar disks, respectively [blue and red shaded regions correspond to current counterflows and their intensity shows the streamfunction magnitude from low (white) to high (intense color)]. Solid and dashed black curves represent discrete wires with opposite senses of current flow, approximating the current continuum with $N_{\varphi}=6$ global contour levels. (c) Axial magnetic field, $B_{z}$, versus axial position, $z$, calculated from the current continuum in (a),(b) in three ways: analytically using Eqs. (26)-(28) (solid red curve); numerically using COMSOL Multiphysics version 5.5a, modeling the high-permeability cylinder as a perfect magnetic conductor (blue dotted curve); numerically without the high-permeability cylinder and using the Biot-Savart law with $N_{\varphi}=100$ contour levels (dashed green curve). Black lines enclose the regions where the axial field gradient deviates by $5 \%$ (dashed) and $1 \%$ (dash-dot).

identical plates of radius $\rho_{c}=0.45 \mathrm{~m}$, inside a magnetic shield of radius $\rho_{s}=0.5 \mathrm{~m}$ and length $L_{s}=1 \mathrm{~m}$. The field gradient is amplified by a factor of 1.06 at the shield's center. If the shield is lengthened, the field gradient is diminished to 0.81 at the shield's center in the long shield limit, as expected from our analysis in the main body of the text. The design in Fig. 10 generates a uniform field, $B_{z}$, in the $z$ direction and extending between two planes of different sizes, with upper and lower coil sizes $\rho_{c 1}=0.95 \mathrm{~m}$ and $\rho_{c 2}=0.35 \mathrm{~m}$, respectively, inside a magnetic shield of radius $\rho_{s}=1 \mathrm{~m}$ and length $L_{s}=1 \mathrm{~m}$. Since the shield and coils have a relatively wide form factor, the field is highly uniform, with the region of axial field deviation below $1 \%$ extending over more than $70 \%$ of the distance between the planes.
[1] X. Wu, Z. Pagel, B. S. Malek, T. H. Nguyen, F. Zi, D. S. Scheirer, and H. Müller, Gravity surveys using a mobile atom interferometer, Sci. Adv. 5, 9 (2019).

[2] V. Menoret, P. Vermeulen, N. L. Moigne, S. Bonvalot, P. Bouyer, A. Landragin, and B. Desruelle, Gravity measurements below $10^{-} 9 \mathrm{~g}$ with a transportable absolute quantum gravimeter, Sci. Rep. 8, 1 (2018).

[3] M. J. Snadden, J. M. McGuirk, P. Bouyer, K. G. Haritos, and M. A. Kasevich, Measurement of the Earth's Gravity Gradient with an Atom Interferometer-Based Gravity Gradiometer, Phys. Rev. Lett. 81, 971 (1998).

[4] K. Bongs, M. Holynski, J. Vovrosh, P. Bouyer, G. Condon, E. Rasel, C. Schubert, W. P. Schleich, and A. Roura, Taking atom interferometric quantum sensors from the 
laboratory to real-world applications, Nat. Rev. Phys. 1, 731 (2019).

[5] V. K. Shah and R. T. Wakai, A compact, high performance atomic magnetometer for biomedical applications, Phys. Med. Biol. 58, 8153 (2013).

[6] E. Boto, N. Holmes, J. Leggett, G. Roberts, V. Shah, S. S. Meyer, L. D. Muñoz, K. J. Mullinger, T. M. Tierney, S. Bestmann, G. R. Barnes, R. Bowtell, and M. J. Brookes, Moving magnetoencephalography towards realworld applications with a wearable system, Nature 555, 657 (2018).

[7] R. M. Hill, E. Boto, M. Rea, N. Holmes, J. Leggett, L. A. Coles, M. Papastavrou, S. K. Everton, B. A. E. Hunt, D. Sims, J. Osborne, V. Shah, R. Bowtell, and M. J. Brookes, Multi-channel whole-head OPM-MEG: Helmet design and a comparison with a conventional system, NeuroImage 219 , 116995 (2020).

[8] T. M. Tierney, N. Holmes, S. Mellor, J. D. López, G. Roberts, R. M. Hill, E. Boto, J. Leggett, V. Shah, M. J. Brookes, R. Bowtell, and G. R. Barnes, Optically pumped magnetometers: From quantum origins to multi-channel magnetoencephalography, NeuroImage 199, 598 (2019).

[9] A. Borna, T. R. Carter, A. P. Colombo, Y.-Y. Jau, J. McKay, M. Weisend, S. Taulu, J. M. Stephen, and P. D. D. Schwindt, Non-invasive functional-brain-imaging with an OPM-based magnetoencephalography system, PLoS ONE 15, 1 (2020).

[10] H. Eswaran, D. Escalona-Vargas, E. H. Bolin, J. D. Wilson, and C. L. Lowery, Fetal magnetocardiography using optically pumped magnetometers: A more adaptable and less expensive alternative? Prenat Diagn. 37, 193 (2017).

[11] S. Lew, M. S. Hämäläinen, and Y. Okada, Toward noninvasive monitoring of ongoing electrical activity of human uterus and fetal heart and brain, Clin. Neurophysiol. 128, 2470 (2017).

[12] S. Strand, W. Lutter, J. F. Strasburger, V.k. Shah, O. Baffa, and R. T. Wakai, Low-cost fetal magnetocardiography: A comparison of superconducting quantum interference device and optically pumped magnetometers, J. Am. Heart. Assoc. 8, 16 (2019).

[13] L. Liu, et al., In-orbit operation of an atomic clock based on laser-cooled ${ }^{87} \mathrm{Rb}$ atoms, Nat. Commun. 9, 16 (2018).

[14] T. E. Chupp, P. Fierlinger, M. J. Ramsey-Musolf, and J. T. Singh, Electric dipole moments of atoms, molecules, nuclei, and particles, Rev. Mod. Phys. 91, 015001 (2019).

[15] I. Altarev, et al., A magnetically shielded room with ultra low residual field and gradient, Rev. Sci. Instrum. 85, 075106 (2014).

[16] J. Li, W. Quan, B. Han, Z. Wang, and J. Fang, Design and optimization of multilayer cylindrical magnetic shield for SERF atomic magnetometer application, IEEE Sens. J. 20, 1793 (2020).

[17] J. Li, Z. Wang, and W. Quan, Multi-objective optimization of multilayer passive magnetic shield based on genetic algorithm, AIP Adv. 9, 125210 (2019).

[18] S. Celozzi, R. Araneo, and G. Lovat, Appendix B: Magnetic Shielding (John Wiley \& Sons Ltd, New York, 2008).

[19] S. Pissanetzky, Minimum energy MRI gradient coils of general geometry, Meas. Sci. Technol. 3, 7 (1992).
[20] M. Poole and R. Bowtell, Novel gradient coils designed using a boundary element method, Concept Magn. Reson. B 3, 162 (2007).

[21] R. Zettera, A. J. Mäkinen, J. Iivanainen, K. C. J. Zevenhoven, R. J. Ilmoniemi, and L. Parkkonen, Magnetic field modeling with surface currents. Part II. Implementation and usage of bfieldtools, J. Appl. Phys. 128, 063905 (2020).

[22] A. J. Mäkinen, R. Zetter, J. Iivanainen, K. C. J. Zevenhoven, L. Parkkonen, and R. J. Ilmoniemi, Magnetic field modeling with surface currents. Part I. Implementation and usage of bfieldtools, J. Appl. Phys. 128, 063906 (2020).

[23] C.-Y. Liu, T. Andalib, D. C. M. Ostapchuk, and C. P. Bidinosti, Analytic models of magnetically enclosed spherical and solenoidal coils, Nucl. Instrum. Methods Phys. Res. 949, 162837 (2020).

[24] R. Lambert and C. Uphoff, Magnetically shielded solenoid with field of high homogeneity, Rev. Sci. Instrum. 46, 3 (1975).

[25] R. J. Hanson and F. M. Pipkin, Magnetically shielded solenoid with field of high homogeneity, Rev. Sci. Instrum. 36, 2 (1965).

[26] J. D. Jackson, Classical Electrodynamics (Wiley, New York, 1998), 3rd ed.

[27] A. Kordyuk, Magnetic levitation for hard superconductors, J. Appl. Phys. 83, 610 (1985).

[28] Q. Cao, D. Pan, J. Li, Y. Jin, Z. Sun, S. Lin, G. Yang, and L. Li, Optimization of a coil system for generating uniform magnetic fields inside a cubic magnetic shield, Energies 11, 3 (2018).

[29] T. Liu, J. Voigt, Z. Sun, A. Schnabel, K. Grueneberg, I. Fan, and L. Li, in Conference on Precision Electromagnetic Measurements (2018), p. 1.

[30] T. Liu, A. Schnabel, Z. Sun, J. Voigt, and L. Li, Approximate expressions for the magnetic field created by circular coils inside a closed cylindrical shield of finite thickness and permeability, J. Magn. Magn. Mater. 507, 166846 (2020).

[31] N. Holmes, T. M. Tierney, J. Leggett, E. Boto, S. Mellor, G. Roberts, R. M. Hill, V. Shah, G. R. Barnes, M. J. Brookes, and R. Bowtell, Balanced, bi-planar magnetic field and field gradient coils for field compensation in wearable magnetoencephalography, Sci. Rep. 9, 14196 (2019).

[32] R. Turner and R. M. Bowley, Passive screening of switched magnetic field gradients, J. Phys. E Sci. Instrum. 19, 876 (1986).

[33] M. Packer, P. J. Hobson, N. Holmes, J. Leggett, P. Glover, M. J. Brookes, R. Bowtell, and T. M. Fromhold, Optimal Inverse Design of Magnetic Field Profiles in a Magnetically Shielded Cylinder, Phys. Rev. Appl. 14, 054004 (2020).

[34] P. Hammond, Electric and magnetic images, Proc. IEE Part C Monogr. 107, 306 (1960).

[35] W. A. Roshen, Effect of finite thickness of magnetic substrate on planar inductors, IEEE Trans. Magn. 26, 270 (1990).

[36] J. Kennedy and R. Eberhart, in Proceedings of ICNN'95International Conference on Neural Networks (1995), Vol. 4, p. 1942. 
[37] F. Roméo and D. I. Hoult, Magnet field profiling: Analysis and correcting coil design, Magn. Reson. Med. 1, 1 (1984).

[38] http://doi.org/10.5281/zenodo.4442661.

[39] J. R. Corea, A. M. Flynn, B. Lechene, G. Scott, G. D. Reed, P. J. Shin, M. Lustig, and A. C. Ariasb, Screenprinted flexible MRI receive coils, Nat. Commun. 7, 10839 (2016).
[40] T. D. Ngo, A. Kashani, G. Imbalzano, K. T. Q. Nguyen, and D. Hui, Additive manufacturing (3D printing): A review of materials, methods, applications and challenges, Compos. B Eng. 143, 172 (2018).

[41] I. S. Gradshteyn and I. M. Ryzhik, Table of Integrals, Series, and Products Vol. 7 (Academic Press, Amsterdam, 2007), p. 1 